\title{
A higher speed type II blowup for the five dimensional energy critical heat equation
}

\author{
Junichi Harada \\ Faculty of Education and Human Studies, Akita University \\ email: harada-j@math.akita-u.ac.jp
}

June 11, 2019

\begin{abstract}
This paper is concerned with blow-up solutions of the five dimensional energy critical heat equation $u_{t}=\Delta u+|u|^{\frac{4}{3}} u$. A goal of this paper is to show the existence of type II blowup solutions which behave as $\|u(t)\|_{\infty} \sim(T-t)^{-3 k}$ $(k=2,3, \cdots)$. Our solutions are the same one formally derived by Filippas, Herrero and Velázquez [7].
\end{abstract}

Keyword: semilinear heat equation; energy critical; type II blowup; matched asymptotic expansion

\section{Introduction}

This paper is concerned with blowup solutions for the semilinear heat equation.

$$
u_{t}=\Delta u+|u|^{p-1} u \quad \text { in } \mathbb{R}^{n} \times(0, T) .
$$

This problem is a simple model of nonlinear diffusion problems. Various complex and interesting phenomena have been found for this 30 years. Our concern in this paper is a blowup caused by a concentration. A local solvability of this problem is well understood, and a blow up occurs at $t=T$ if $\limsup _{t \rightarrow T}\|u(t)\|_{\infty}=\infty$. For a blowup solution, the blowup is called type I if $\lim _{\sup _{t \rightarrow T}}(T-t)^{\frac{1}{p-1}}\|u(t)\|_{\infty}<\infty$, and type II if $\lim \sup _{t \rightarrow T}(T-t)^{\frac{1}{p-1}}\|u(t)\|_{\infty}=\infty$. A typical type I blowup solution is given by

$$
w(x, t)=(p-1)^{-\frac{1}{p-1}}(T-t)^{-\frac{1}{p-1}} .
$$

In the study of blowup problems, there are two important critical values of $p$ defined by

$$
p_{\mathrm{S}}=\frac{n+2}{n-2}, \quad p_{\mathrm{JL}}= \begin{cases}\infty & \text { if } n \leq 10, \\ 1+\frac{4}{n-4-2 \sqrt{n-1}} & \text { if } n \geq 11 .\end{cases}
$$

For the case $1<p<p_{\mathrm{S}}$, it is well known that every blowup solution is locally approximated by 1.2 , namely type I (see [8]). On the other hand, for the case $p \geq p_{S}$, different types of blowup behavior are observed. A type II blowup solution is first discovered by Herrero and Velázquez [11, 12] (see also [16]). They construct type II blowup solutions with the exact blowup rates for the case $p>p_{\mathrm{JL}}$. Very recently Seki [18] proves the existence of type II blowup solutions for the case $p=p_{\mathrm{JL}}$. In the middle range of $p \in\left(p_{\mathrm{S}}, p_{\mathrm{JL}}\right)$, Matano and Merl [14] exclude the occurrence of a type II blowup under a radial setting. Another example of a type II blowup is found by Filippas, Herrero and Velázquez [7] for the energy critical case $p=p_{\mathrm{S}}$. They formally obtain a type II blowup by using the matched asymptotic expansion approach. The blowup rate of their solutions are given by (the blowup rates for $n=5$ p. 2971 and $n=6$ p. 2972 in $[7]$ seem to be incorrect by a trivial miscalculation)

$$
\|u(t)\|_{\infty} \sim \begin{cases}(T-t)^{-k} & n=3 \\ (T-t)^{-k}|\log (T-t)|^{\frac{2 k}{2 k-1}} & n=4 \\ (T-t)^{-3 k} & n=5 \quad \text { (corrected) } \\ (T-t)^{-\frac{5}{2}}|\log (T-t)|^{\frac{15}{4}} & n=6 \quad \text { (corrected) }\end{cases}
$$

where $k=1,2,3, \cdots$. As for a higher dimensional case $n \geq 7$, the possibility of a type II blowup near the ground states is ruled out by Collot, Merle and Raphaël [1]. The first rigorous proof of the existence of a type II blowup for the energy critical case is given by Schweyer [19. He constructs a type II blowup solution for $n=4$ by adapting the energy method developed in the study of geometrical dispersive problems ([17, 15]) to the problem (1.1). The blowup rate of his solution coincides with $k=1$ in 1.3 . Very recently Cortázar, del Pino and Musso [6] obtain a type II blowup for $n=5$ with the same blowup rate as $k=1$ in $(1.3)$. They apply so-called the inner-outer gluing method developed in [2, 3, 4]. Furthermore a new type of type II blowup not listed in 51.3 is found by del Pino, Musso and Wei 5 ] for $n \geq 7$. In this paper, we prove the existence of type II blowup solutions for $n=5$ with a higher blowup speed. The solutions constructed here give the first example for $k \geq 2$ in $(1.3)$. 


\section{Main result}

Let $\mathrm{Q}_{\lambda}(x)$ be the positive radial stationary solution given by

$$
\mathrm{Q}_{\lambda}(x)=\lambda^{-\frac{n-2}{2}}\left(1+\frac{1}{n(n-2)} \frac{|x|^{2}}{\lambda^{2}}\right)^{-\frac{n-2}{2}} \quad \text { (ground state). }
$$

Theorem 1. Let $n=5$ and $p=p_{S}$. For any integer $l \geq 1$ and any two constants $A>0, \kappa \in(0,1)$, there exist $T>0$ and a radial solution $u(x, t) \in C\left(\mathbb{R}^{5} \times[0, T)\right) \cap C^{2,1}\left(\mathbb{R}^{5} \times(0, T)\right)$ of (1.1) such that

$$
u(x, t)=\mathrm{Q}_{\lambda(t)}(x)+v(x, t),
$$

where $\lambda(t)$ and $v(x, t)$ satisfy

$$
\left|\lambda(t)-A(T-t)^{2 l+2}\right|<\kappa A(T-t)^{2 l+2} \quad \text { and } \quad v(x, t) \in L^{\infty}\left(\mathbb{R}^{5} \times(0, T)\right) .
$$

Remark 1. The blowup rate of the solution obtained in Theorem 1 is given by

$$
\|u(t)\|_{\infty} \sim\left\|\mathrm{Q}_{\lambda(t)}\right\|_{\infty} \sim \lambda(t)^{-\frac{n-2}{2}} \sim(T-t)^{-3(l+1)} \quad(l=1,2,3, \cdots) .
$$

This solution gives an example for $k=l+1 \geq 2$ in $(1.3)$.

Remark 2. Our strategy is based on so-called the inner-outer gluing method used in [6]. We look for a solution of the form

$$
u(x, t)=\mathrm{Q}_{\lambda(t)}+\underbrace{\Theta(x, t)+\lambda^{-\frac{n-2}{2}} \epsilon(y, t)+w(x, t)}_{=v(x, t)}, \quad y=\frac{x}{\lambda} .
$$

The function $\Theta(x, t)$ is a particular solution of $\Theta_{t}=\Delta_{x} \Theta$. In [6], they consider a simpler case, where $\Theta(x, t)$ is chosen to be a constant function. In this paper, we try other types of a particular solution $\Theta(x, t)$ satisfying $\Theta(x, t) \sim(T-t)^{l}$ for $|x| \sim \sqrt{T-t}$. This contributes to the blowup rate. Generally the function $\Theta(x, t)$ can not be chosen arbitrarily, since it must satisfy a certain matching condition (see Section 4). The functions $\epsilon(y, t)$ and $w(x, t)$ describe the behavior in the inner region $|x| \sim \lambda(t)$ and in the self-similar region $|x| \sim \sqrt{T-t}$ respectively. Since the behavior of the inner solution $\epsilon(y, t)$ is almost the same as that of [G], it can be treated in the same manner. A main part of this paper is to handle the outer solution $w(x, t)$. To derive an appropriate decay estimate of $w(x, t)$, we borrow techniques from [11, 12] (see also [16, 18]), where they treat a different type of outer solutions.

\section{Preliminary}

Throughout this paper, $\chi(\xi) \in C^{\infty}(\mathbb{R})$ stands for a standard cut off function satisfying

$$
\chi(\xi)= \begin{cases}1 & \text { if } \xi<1 \\ 0 & \text { if } \xi>2\end{cases}
$$

Furthermore we write

$$
f(u)=|u|^{p-1} u, \quad p=\frac{n+2}{n-2} .
$$

\subsection{Linearization aound the ground state}

Let us consider the eigenvalue problem related to a linearization around the ground state $\mathrm{Q}(y)=\left.\mathrm{Q}_{\lambda}(y)\right|_{\lambda=1}$.

$$
-H_{y} \psi=\mu \psi \quad \text { in } \mathbb{R}^{n}
$$

where the operator $H_{y}$ is define by

$$
H_{y}=\Delta_{y}+V(y), \quad V(y)=f^{\prime}(\mathrm{Q}(y))=p \mathrm{Q}(y)^{p-1} .
$$

We recall that the operator $H_{y}$ has a negative eigenvalue $\mu_{1}<0$ and a zero eigenvalue. We denote by $\psi_{1}(r)$ a positive radial eigenfunction associated to the negative eigenvalue with $\psi_{1}(0)=1$. Furthermore there exists $C>0$ such that

$$
\psi_{1}(r)<C(1+r)^{-\frac{n-1}{2}} e^{-\sqrt{\left|\mu_{1}\right|} r} .
$$

The eigenfunction associated to a zero eigenvalue is explicitly given by

$$
\Lambda_{y} \mathrm{Q}(y)=\left(\frac{n-2}{2}+y \cdot \nabla_{y}\right) \mathrm{Q}(y) .
$$




\subsection{Purterbated linearized problem}

We next consider the eigenvalue problem 3.1 in a bounded but very large domain.

$$
\begin{cases}-H_{y} \psi=\mu \psi & \text { in } B_{R} \\ \psi=0 & \text { on } \partial B_{R} \\ \psi \text { is raidal. } & \end{cases}
$$

We denote the $i$ th eigenvalue of $(3.2)$ by $\mu_{i}^{(R)}$ and the associated eigenfunction by $\psi_{i}^{(R)}$. We normalize $\psi_{i}^{(R)}(r)$ as $\psi_{i}^{(R)}(0)=1$. Most of the lemmas stated in this subsection are proved in Section 7 [2] However for the sake of convenience, we give the proofs. Throughout this subsection, we write

$$
k_{1} \lesssim k_{2} \quad\left(k_{1}, k_{2}>0\right)
$$

if there is a universal constant $c>0$ independent of $R$ such that $k_{1}<c k_{2}$. This definitions will be changed slightly in Section 5.3 .

Lemma 3.1. It holds that for any $R>0$

$$
0<\psi_{1}^{(R)}(r) \lesssim(1+r)^{-\frac{n-1}{2}} e^{-\sqrt{\left|\mu_{1}\right|} r} \quad \text { for } r \in(0, R) .
$$

Proof. It is enough to prove $\psi_{1}^{(R)}(r)<2 \psi_{1}(r)$. We prove by contradiction. Suppose that there exists $r_{1} \in(0, R)$ such that $\psi_{1}^{(R)}(r)<2 \psi_{1}(r)$ for $r<r_{1}$ and $\psi_{1}^{(R)}\left(r_{1}\right)=2 \psi_{1}\left(r_{1}\right)$. We now define $\psi_{0} \in H_{0}^{1}\left(B_{R}\right)$ as

$$
\psi_{0}(r)= \begin{cases}2 \psi_{1}(r)-\psi_{1}^{(R)}(r) & \text { for } r<r_{1}, \\ 0 & \text { for } r_{1}<r<R .\end{cases}
$$

By the monotonic dependence of the eigenvalue with respect to the domain, it holds that $\mu_{1}^{(R)}>\mu_{1}$. Therefore we get

$$
\begin{aligned}
\int_{B_{R}}\left(\left|\nabla_{y} \psi_{0}\right|^{2}-V \psi_{0}^{2}\right) d y & =\mu_{1}^{(R)} \int_{B_{R}} \psi_{0}^{2} d y+\left(\mu_{1}-\mu_{1}^{(R)}\right) \int_{B_{R}} 2 \psi_{1} \psi_{0} d y \\
& <\mu_{1}^{(R)} \int_{B_{R}} \psi_{0}^{2} d y
\end{aligned}
$$

However this contradicts to characterization of $\mu_{1}^{(R)}$. The proof is completed.

Lemma 3.2. There exists $R_{1}>0$ such that if $R>R_{1}$

$$
\left|\psi_{2}^{(R)}(r)\right| \lesssim(1+r)^{-(n-2)} \quad \text { for } r \in(0, R)
$$

Proof. Put $\psi_{2}(r)=\Lambda_{y} \mathrm{Q}(r) / \Lambda_{y} \mathrm{Q}(0)$. It is clear that $\psi_{2}$ gives the eigenfunction of (3.1) associated to a zero eigenvalue and $\psi_{2}(0)=1$. Let $r_{0}$ and $r_{0}^{(R)}$ be the unique zero of $\psi_{2}(r)$ and $\psi_{2}^{(R)}$ respectively. The Sturm comparison principle implies that $\mu_{2}^{(R)}>0$ and $r_{0}^{(R)}<r_{0}$. We easily see that $\lim _{R \rightarrow \infty} \mu_{2}^{(R)}=0$ and $\lim _{R \rightarrow \infty} \psi_{2}^{(R)}(r)=\psi_{2}(r)$ locally uniformly in $r \in[0, \infty)$. Therefore there exists $R_{1}>0$ such that $\psi_{2}\left(r_{0}+1\right)<\frac{1}{2} \psi_{2}^{(R)}\left(r_{0}+1\right)<0$ if $R>R_{1}$. We now define $r_{1}>r_{0}$ as

$$
r_{1}=\sup \left\{r \in\left(r_{0}, r_{0}+1\right) ; \psi_{2}(r)=\frac{1}{2} \psi_{2}^{(R)}(r)\right\} \text {. }
$$

From this definition, we immediately see that $\psi_{2}\left(r_{1}\right)=\frac{1}{2} \psi_{2}^{(R)}\left(r_{1}\right)$ and

$$
\psi_{2}(r)<\frac{1}{2} \psi_{2}^{(R)}(r) \quad \text { for } r_{1}<r<r_{0}+1
$$

We now suppose that there exists $r_{2}>r_{1}$ such that $\psi_{2}\left(r_{2}\right)=\frac{1}{2} \psi_{2}^{(R)}\left(r_{2}\right)$ and

$$
\psi_{2}(r)<\frac{1}{2} \psi_{2}^{(R)}(r) \quad \text { for } r_{1}<r<r_{2} .
$$

However this contradicts the Sturm comparison principle. Therefore we obtain

$$
\psi_{2}(r)<\frac{1}{2} \psi_{2}^{(R)}(r)<0 \quad \text { for } r_{0}+1<r<R .
$$

Since $\left|\psi_{2}(r)\right| \lesssim(1+r)^{-(n-2)}$, we complete the proof. 
Lemma 3.3 (Lemma $7.2[2]$ ). Let $n \geq 5$. There exists $R_{2}>0$ such that if $R>R_{2}$

$$
\mu_{2}^{(R)} \gtrsim R^{-(n-2)}
$$

Proof. We recall that $Z_{1}(r)=\Lambda_{y} \mathrm{Q}(r)$ gives a solution of $H_{y} Z=0$. Let $Z_{2}(r)=\Gamma(r)$ be another independent solution of $H_{y} Z=0$. Since $\psi_{2}^{(R)}(r)$ is a solution of $(3.2)$ with $\mu=\mu_{2}^{(R)}$, it is expressed as

$$
\begin{aligned}
\psi_{2}^{(R)}(r)= & k Z_{2}(r) \int_{0}^{r} \mu_{2}^{(R)} \psi_{2}^{(R)}\left(r^{\prime}\right) Z_{1}\left(r^{\prime}\right) r^{\prime n-1} d r^{\prime}+k Z_{1}(r) \int_{r}^{R} \mu_{2}^{(R)} \psi_{2}^{(R)}\left(r^{\prime}\right) Z_{2}\left(r^{\prime}\right) r^{\prime n-1} d r^{\prime} \\
& \quad-k \frac{Z_{2}(R)}{Z_{1}(R)} Z_{1}(r) \int_{0}^{R} \mu_{2}^{(R)} \psi_{2}^{(R)}\left(r^{\prime}\right) Z_{1}\left(r^{\prime}\right) r^{\prime n-1} d r^{\prime} \\
=: & \mu_{2}^{(R)}\left(A_{1}+A_{2}-A_{3}\right),
\end{aligned}
$$

where $k$ is a constant depending on $Z_{1}(r)$ and $Z_{2}(r)$. Since $\left|Z_{2}(r)\right| \lesssim 1+r^{-(n-2)}$, we easily see that

$$
\begin{aligned}
\left\|A_{1}\right\|_{L^{2}\left(B_{R}\right)} & \lesssim\left\|\psi_{2}^{(R)}\right\|_{L^{\infty}\left(B_{1}\right)}\left\|Z_{1}\right\|_{L^{\infty}\left(B_{1}\right)}+R^{\frac{n}{2}}\left\|\psi_{2}^{(R)}\right\|_{L^{2}\left(B_{R}\right)}\left\|Z_{1}\right\|_{L^{2}\left(B_{R}\right)}, \\
\left\|A_{2}\right\|_{L^{2}\left(B_{R}\right)} & \lesssim\left\|Z_{1}\right\|_{L^{2}\left(B_{R}\right)}\left\|\psi_{2}^{(R)}\right\|_{L^{\infty}\left(B_{1}\right)}+R^{\frac{n}{2}}\left\|Z_{1}\right\|_{L^{2}\left(B_{R}\right)}\left\|\psi_{2}^{(R)}\right\|_{L^{2}\left(B_{R}\right)}, \\
\left\|A_{3}\right\|_{L^{2}\left(B_{R}\right)} & \lesssim\left|\frac{Z_{2}(R)}{Z_{1}(R)}\right|\left\|Z_{1}\right\|_{L^{2}\left(B_{R}\right)}^{2}\left\|\psi_{2}^{(R)}\right\|_{L^{2}\left(B_{R}\right)} .
\end{aligned}
$$

Since $\lim _{R \rightarrow \infty} \psi_{2}^{(R)}(r)=\psi_{2}(r)$ uniformly in $r \in[0,1]$ (see Lemma 3.2 , it follows that $\left\|\psi_{2}^{(R)}\right\|_{L^{\infty}\left(B_{1}\right)} \lesssim 1$. Therefore when $n \geq 5$, we get from Lemma 3.2 that

$$
\left\|\psi_{2}^{(R)}\right\|_{L^{2}\left(B_{R}\right)} \lesssim \mu_{2}^{(R)}\left(1+R^{\frac{n}{2}}+R^{n-2}\right)
$$

Since $\lim _{R \rightarrow \infty}\left\|\psi_{2}^{(R)}\right\|_{L^{2}\left(B_{R}\right)}=\left\|\Lambda_{y} \mathrm{Q}\right\|_{L_{2}\left(\mathbb{R}^{n}\right)}$ if $n \geq 5$ (see Lemma 3.2, we complete the proof.

\subsection{Behavior of the Laplace equation wiht a purterbation term}

Consider a radial solution of

$$
\Delta_{y} p+\left(1-\chi_{M}\right) V(y) p=0 \quad \text { in } \mathbb{R}^{n},
$$

where $\chi_{M}(y)=\chi\left(\frac{|y|}{M}\right)$. Let $p_{M}(r)$ be a radial solution of this problem satisfying $p_{M}(r)=1$ for $r<M$.

Lemma 3.4 (see proof of Lemma 7.3 [2]). There exist $k \in(0,1)$ and $M_{1}>0$ such that if $M>M_{1}$

$$
k<p_{M}(r) \leq 1 \quad \text { for } r \in(0, \infty) .
$$

Proof. Since $V(r) \sim \frac{1}{r^{4}}$ for $r>1$, we can take $M_{1}>0$ such that

$$
V(r)<\frac{n-3}{r^{2}} \quad \text { for } r>M_{1}
$$

Let $\bar{p}(r)=\frac{M_{1}^{n-3}}{r^{n-3}}$. It satisfies

$$
\Delta_{y} \bar{p}-\frac{n-3}{r^{2}} \bar{p}=0
$$

Therefore by the Sturm comparison principle, it holds that

$$
p_{M_{1}}(r)>\bar{p}(r)=\frac{M_{1}^{n-3}}{r^{n-3}} \quad \text { for } r>M_{1} .
$$

Let $Z_{1}(r)$ and $Z_{2}(r)$ be given in the proof of Lemma 3.3 . Since $p_{M_{1}}(r)$ satisfies $H_{y} p_{M_{1}}=0$ for $r>2 M_{1}$, there exist two constants $c_{1}, c_{2} \in \mathbb{R}$ such that $p_{M_{1}}(r)=c_{1} Z_{1}(r)+c_{2} Z_{2}(r)$ for $r>2 M_{1}$. We recall that $\lim _{r \rightarrow \infty} Z_{2}(r)=\alpha \neq 0$. Since $p_{M_{1}}(r)$ satisfies (3.3), $c_{2} \alpha$ must be positive. Therefore it holds that $k:=\inf _{r>0} p_{M_{1}}(r)>0$. For the case $M>M_{1}$, by the Sturm comparison principle, we conclude $p_{M}(r) \geq p_{M_{1}}(r) \geq k$ for $r>0$. Since $p_{M}(r)$ is positive, we easily check that $\partial_{r} p_{M}(r) \leq 0$, which implies $p_{M}(r)<1$. The proof is completed. 


\subsection{The Schauder estimate for parabolic equations}

Put $Q=B_{1} \times(0,1)$. For $\alpha \in(0,1)$, we define the Hölder norm.

$$
\|u\|_{C^{\alpha}(Q)}:=\|u\|_{L^{\infty}(Q)}+[u]_{C^{\alpha}(Q)}, \quad[u]_{C^{\alpha}(Q)}:=\sup _{\left(x_{1}, t_{1}\right),\left(x_{2}, t_{2}\right) \in Q} \frac{\left|u\left(x_{1}, t_{1}\right)-u\left(x_{2}, t_{2}\right)\right|}{\left|x_{1}-x_{2}\right|^{\alpha}+\left|t_{1}-t_{2}\right|^{\frac{\alpha}{2}}} .
$$

We recall the local Höder estimate for parabolic equations.

Lemma 3.5 (Theorem 4.8 p. $56[13]$ ). Let $\alpha \in(0,1)$ and $V(x, t) \in L^{\infty}(Q)$ satisfy $\|V\|_{L^{\infty}(Q)}<M$. There exists $c_{M}>0$ such that if $u(x, t), \nabla_{x} u(x, t) \in C^{\alpha}(Q)$ and $u(x, t)$ satisfies

$$
u_{t}=\Delta_{x} u+V(x, t) u+f(x, t) \quad \text { in } B_{1} \times(0,1),
$$

then

$$
\|u\|_{C^{\alpha}\left(B_{\frac{1}{2}} \times\left(\frac{1}{2}, 1\right)\right)}+\left\|\nabla_{x} u\right\|_{C^{\alpha}\left(B_{\frac{1}{2}} \times\left(\frac{1}{2}, 1\right)\right)}<c_{M}\left(\|u\|_{L^{\infty}(Q)}+\|f\|_{L^{\infty}(Q)}\right)
$$

\subsection{Local behavior of the heat equation}

Consider the heat equation

$$
u_{t}=\Delta u \quad \text { in } \mathbb{R}^{n} \times(0, \infty) .
$$

To describe the local behavior of solutions, we use self-similar variables.

$$
\theta(z, \tau)=u(x, t), \quad z=\frac{x}{\sqrt{1-t}}, \quad 1-t=e^{-\tau} .
$$

This function $\theta(z, \tau)$ solves

$$
\theta_{\tau}=A_{z} \theta \quad \text { in } \mathbb{R}^{n} \times(0, \infty)
$$

where $A_{z}=\Delta_{z}-\frac{z}{2} \cdot \nabla_{z}$. We define the associated wighted $L^{2}$ space by

$$
L_{\rho}^{2}\left(\mathbb{R}^{n}\right):=\left\{f \in L_{\mathrm{loc}}^{2}\left(\mathbb{R}^{n}\right) ;\|f\|_{\rho}<\infty\right\}, \quad\|f\|_{\rho}^{2}=\int_{\mathbb{R}^{n}} f(z)^{2} \rho(z) d z, \quad \rho(z)=e^{-\frac{|z|^{2}}{4}} .
$$

The inner product is denoted by

$$
\left(f_{1}, f_{2}\right)_{\rho}==\int_{\mathbb{R}^{n}} f_{1}(z) f_{2}(z) \rho(z) d z .
$$

Consider the eigenvalue problem

$$
-\Delta e_{i}+\frac{z}{2} \cdot e_{i}=\lambda_{l} e_{i} \quad \text { in } L_{\rho, \mathrm{rad}}^{2}\left(\mathbb{R}^{n}\right)
$$

It is known that

- $\lambda_{i}=i \quad(i=0,1,2, \cdots)$ and

- $e_{i}(z) \in L_{\rho, \text { rad }}^{2}\left(\mathbb{R}^{n}\right)$ is the $2 l$ th-degree polynomial.

We normalize the eigenfunction $e_{i}(z)$ as $e_{i}(0)=1$, which implies

$$
e_{i}(z)=1+a_{1}|z|^{2}+a_{2}|z|^{4}+\cdots+a_{l}|z|^{2 l} .
$$

The function

$$
\Theta_{i}(x, t)=A(T-t)^{i} e_{i}(z), \quad z=\frac{x}{\sqrt{T-t}} \quad(i=0,1,2, \cdots)
$$

gives a solution of (3.4). This function plays a crucial role in our argument. The constant $A$ is chosen to be $A=-1$ later. We denote by $e^{A_{z} \tau} \theta_{0}$ a solution of (3.5) with the initial date $\theta_{0}$ for $\tau=0$. This is expressed by

$$
e^{A_{z} \tau} \theta_{0}=\frac{c_{n}}{\left(1-e^{-\tau}\right)^{\frac{n}{2}}} \int_{\mathbb{R}^{n}} e^{\frac{\left|e^{-\frac{\tau}{2}} z-\xi\right|^{2}}{4\left(1-e^{-\tau}\right)}} \theta_{0}(\xi) d \xi
$$

By using this formula, we can obtain the following parabolic estimate for (3.5) (see proof of Lemma 2.2 in [10]).

Lemma 3.6. There exists $C>0$ such that

$$
\left|\left(e^{A_{z} \tau} \theta_{0}\right)(z)\right|<C \frac{e^{\frac{e^{-\tau}|z|^{2}}{4\left(1+e^{-\tau}\right)}}}{\left(1-e^{-\tau}\right)^{\frac{n}{4}}}\left\|\theta_{0}\right\|_{\rho} \quad \text { for }(z, \tau) \in \mathbb{R}^{n} \times(0, \infty) .
$$


We prepare another type of parabolic estimates given in $[9]$.

Lemma 3.7 (Lemma 2.2 in [9])). For any $l \in \mathbb{N}$, there exists $C_{l}>0$ such that if $\theta_{0}=|z|^{2 l}$

$$
\left|\left(e^{A_{z} \tau} \theta_{0}\right)(z)\right|<C_{l}\left(1+e^{-l \tau}|z|^{2 l}\right) \quad \text { for }(z, \tau) \in \mathbb{R}^{n} \times(0, \infty) .
$$

We next consider the nonhomogeneous heat equation.

$$
\left\{\begin{array}{ll}
\Phi_{\tau}=A_{z} \Phi+\frac{e^{-\tau}}{\lambda(\tau)^{2}} \frac{1}{1+|y|^{2+a}} & \text { in } \mathbb{R}^{n} \times\left(\tau_{1}, \infty\right), \\
\Phi=0 & \text { for } \tau=\tau_{1},
\end{array} \quad y=\frac{e^{-\frac{\tau}{2}}}{\lambda(\tau)} z .\right.
$$

Lemma 3.8 (Lemma $4.2 \mathrm{p} 8$ [6]). Let $a>0, \gamma>\frac{1}{2}$ and $T_{1}=e^{-\tau_{1}}$. If $\lambda(\tau)$ satisfies

$$
k_{1} e^{-\gamma \tau}<\lambda<k_{2} e^{-\gamma \tau} \quad \text { and } \quad\left|\frac{d \lambda}{d \tau}\right|<k_{3} \lambda
$$

there exists $C>0$ depending on $a, \gamma, k_{1}, k_{2}, k_{3}$ such that a solution $\Phi(z, \tau)$ of (3.7) satisfies

$$
|\Phi(z, \tau)|<C\left(\frac{1}{1+|y|^{a}}+T_{1}^{\left(\gamma-\frac{1}{2}\right) a}\right), \quad y=\frac{e^{-\frac{\tau}{2}}}{\lambda} z .
$$

Proof. We change variable.

$$
\psi(x, t)=\Phi\left(e^{\frac{\tau}{2}} x, \tau\right), \quad T_{1}-t=-e^{-\tau} .
$$

The function $\psi(x, t)$ solves

$$
\left\{\begin{array}{ll}
\psi_{t}=\Delta_{x} \psi+\frac{1}{\lambda^{2}} \frac{1}{1+|y|^{2+a}} & \text { in } \mathbb{R}^{n} \times\left(0, T_{1}\right), \\
\psi=0 & \text { for } t=0,
\end{array} \quad y=\frac{x}{\lambda} .\right.
$$

From Lemma 2.2 in [6], there exists $C>0$ such that

$$
|\psi(x, t)|<C\left(\frac{1}{1+|y|^{a}}+T_{1}^{\left(\gamma-\frac{1}{2}\right) a}\right)
$$

The proof is completed.

\section{Formal derivation of blowup speed}

We fix $l \in \mathbb{N}$. Throughout this paper, we write (see 3.6 )

$$
\Theta(x, t)=\Theta_{l}(x, t)=A(T-t)^{l} e_{l}(z), \quad z=\frac{x}{\sqrt{T-t}} .
$$

We look for solutions of the form

$$
u(x, t)=\mathrm{Q}_{\lambda(t)}(x)+\Theta(x, t)+v(x, t),
$$

where $v(x, t)$ is a remainder term. A function $v(x, t)$ satisfies

$$
v_{t}=\Delta v+f^{\prime}\left(\mathrm{Q}_{\lambda}\right)(\Theta+v)+\mathrm{N}(v)+\frac{\lambda_{t}}{\lambda^{\frac{n}{2}}} \Lambda_{y} \mathrm{Q}(y), \quad y=\frac{x}{\lambda} .
$$

The nonlinear term $\mathbf{N}(v)$ is defined by

$$
\mathrm{N}(v)=f\left(\mathrm{Q}_{\lambda}+\Theta+v\right)-f\left(\mathrm{Q}_{\lambda}\right)-f^{\prime}\left(\mathrm{Q}_{\lambda}\right)(\Theta+v) .
$$

We write $v(x, t)$ as

$$
v(x, t)=\lambda^{-\frac{n-2}{2}} \epsilon(y, t), \quad y=\frac{x}{\lambda} .
$$

The relation (4.1) is rewritten as

$$
u(x, t)=\mathrm{Q}_{\lambda(t)}(x)+\Theta(x, t)+\epsilon_{\lambda(t)}(x, t) .
$$


Under this setting, it is natural to assume that

$$
|\epsilon(y, s)| \ll \mathbf{Q}(y)
$$

The function $\epsilon(y, t)$ satisfies

$$
\frac{\epsilon_{t}}{\lambda^{\frac{n-2}{2}}}=\frac{H_{y} \epsilon}{\lambda^{\frac{n+2}{2}}}+\frac{V(y)}{\lambda^{2}} \Theta(x, t)+\mathrm{N}(v)+\frac{\lambda_{t}}{\lambda^{\frac{n}{2}}} \Lambda_{y} \mathrm{Q}(y)+\frac{\lambda_{t}}{\lambda^{\frac{n}{2}}} \Lambda_{y} \epsilon .
$$

Neglecting $\mathrm{N}(v)$ and assuming 4.2 , we obtain

$$
\lambda^{2} \epsilon_{t} \sim H_{y} \epsilon+\lambda^{\frac{n-2}{2}} V(y) \Theta(x, t)+\lambda \lambda_{t} \Lambda_{y} \mathrm{Q}(y) .
$$

Since $x=\lambda(t) y$ and $\lim _{t \rightarrow T} \lambda(t)=0$, we here replace $\Theta(x, t)$ by $\Theta(0, t)$.

$$
\lambda^{2} \epsilon_{t} \sim H_{y} \epsilon+\lambda^{\frac{n-2}{2}} V(y) \Theta(0, t)+\lambda \lambda_{t} \Lambda_{y} \mathrm{Q}(y)
$$

We take the inner product $\left(\cdot, \Lambda_{y} \mathrm{Q}\right)_{L_{y}^{2}\left(\mathbb{R}^{n}\right)}$ to get

$$
\lambda^{2}\left(\epsilon_{t}, \Lambda_{y} \mathrm{Q}\right)_{2} \sim \lambda^{\frac{n-2}{2}} \Theta(0, t)\left(V, \Lambda_{y} \mathrm{Q}\right)_{2}+\lambda \lambda_{t}\left\|\Lambda_{y} \mathrm{Q}\right\|_{2}^{2}
$$

In addition to 4.2 , we assume that the left-hand side is negligible in the relation. Since $\Theta(0, t)=A(T-t)^{l}$, we obtain

$$
0 \sim \lambda^{\frac{n-2}{2}} A(T-t)^{l}\left(V, \Lambda_{y} \mathrm{Q}\right)_{2}+\lambda \lambda_{t}\left\|\Lambda_{y} \mathrm{Q}\right\|_{2}^{2}
$$

By a direct calculation, the first term is computed as

$$
\begin{aligned}
\left(V, \Lambda_{y} \mathrm{Q}\right)_{2} & =\left(p \mathrm{Q}^{p-1}, \Lambda_{y} \mathrm{Q}\right)_{2}=\left.\frac{d}{d \lambda} \int_{\mathbb{R}^{n}} \mathrm{Q}_{\frac{1}{\lambda}}^{p} d y\right|_{\lambda=1} \\
& =\left.\frac{d}{d \lambda} \lambda^{-\frac{n-2}{2}}\|\mathrm{Q}\|_{p}^{p}\right|_{\lambda=1}=-\frac{n-2}{2}\|\mathrm{Q}\|_{p}^{p}
\end{aligned}
$$

Therefore we obtain a differential equation for $\lambda(t)$.

$$
\frac{\lambda_{t}}{\lambda^{\frac{n-4}{2}}} \sim \frac{A(n-2)}{2} \frac{\|\mathrm{Q}\|_{p}^{p}}{\left\|\Lambda_{y} \mathrm{Q}\right\|_{2}^{2}}(T-t)^{l}
$$

Since $\lambda(t)$ must be positive and $\lim _{t \rightarrow T} \lambda(t)=0$, the constant $A$ must be negative. We finally obtain

$$
\lambda(t) \sim\left(\frac{-A(6-n)(n-2)}{4(l+1)} \frac{\|\mathrm{Q}\|_{p}^{p}}{\left\|\Lambda_{y} \mathrm{Q}\right\|_{2}^{2}}\right)^{\frac{2}{6-n}}(T-t)^{\frac{2(l+1)}{6-n}}
$$

This is the desired result. From now on, we choose

$$
A=-1
$$

\section{Formulation}

In this section, we set up our problem as in the proof of Theorem 1 [6]. To justify the argument in Section 4 , we need several corrections.

\subsection{Setting}

We look for solutions of the form

$$
u(x, t)=\mathrm{Q}_{\lambda(t)}(x)+\Theta(x, t) \chi_{\mathrm{out}}+v(x, t),
$$

where $\chi_{\text {out }}$ is a cut off function defined by

$$
\chi_{\mathrm{out}}=\chi\left((T-t)^{B}|z|\right), \quad z=\frac{x}{\sqrt{T-t}}, \quad B=\frac{l+\frac{1}{2}}{2 l+2} .
$$

A function $v(x, t)$ satisfies

$$
v_{t}=\Delta v+f^{\prime}\left(\mathrm{Q}_{\lambda}\right)\left(\Theta \chi_{\mathrm{out}}+v\right)+\mathrm{N}(v)+\frac{\lambda_{t}}{\lambda^{\frac{n}{2}}} \Lambda_{y} \mathrm{Q}(y)+h_{\mathrm{out}},
$$


where

$$
h_{\text {out }}=2 \nabla_{x} \Theta \cdot \nabla_{x} \chi_{\text {out }}+\Theta \Delta_{x} \chi_{\text {out }}-\Theta \partial_{t} \chi_{\text {out }}
$$

We decompose $v(x, t)$ as

$$
v(x, t)=\lambda^{-\frac{n-2}{2}} \epsilon(y, t) \chi_{\mathrm{in}}+w(x, t), \quad y=\frac{x}{\lambda} .
$$

The function $\epsilon(y, t)$ is defined on $(y, t) \in B_{2 R} \times(0, T)$ and $\chi_{\text {in }}=\chi\left(\frac{|y|}{R}\right)$. Plugging this into (5.1), we get

$$
\frac{\epsilon_{t}}{\lambda^{\frac{n-2}{2}}} \chi_{\mathrm{in}}+w_{t}=\frac{H_{y} \epsilon}{\lambda^{\frac{n+2}{2}}} \chi_{\mathrm{in}}+\Delta w+\frac{V(y)}{\lambda^{2}}\left(\Theta \chi_{\mathrm{out}}+v\right)+\mathrm{N}(v)+\frac{\lambda_{t}}{\lambda^{\frac{n}{2}}} \Lambda_{y} \mathrm{Q}(y)+h_{\mathrm{out}}+h_{\mathrm{in}},
$$

where

$$
h_{\mathrm{in}}=\frac{1}{\lambda^{\frac{n+2}{2}}}\left(2 \nabla_{y} \epsilon \cdot \nabla_{y} \chi_{\mathrm{in}}+\epsilon \Delta_{y} \chi_{\mathrm{in}}\right)+\frac{\lambda_{t}}{\lambda^{\frac{n}{2}}} \Lambda_{y} \epsilon \chi_{\mathrm{in}}-\frac{1}{\lambda^{\frac{n-2}{2}}} \epsilon \partial_{t} \chi_{\mathrm{in}} .
$$

We introduce a parabolic system of $(\epsilon(y, t), w(x, t))$.

$$
\begin{cases}\lambda^{2} \epsilon_{t}=H_{y} \epsilon+G_{\text {in }}(\lambda, w) & \text { in } B_{2 R} \times(0, T), \\ w_{t}=\Delta_{x} w+G_{\text {out }}(\lambda, w, \epsilon) & \text { in } \mathbb{R}^{5} \times(0, T),\end{cases}
$$

where

$$
\begin{aligned}
G_{\text {in }}(\lambda, w) & =\lambda^{\frac{n-2}{2}} \Theta(x, t) V+\lambda^{\frac{n-2}{2}} w(x, t) V+\lambda \lambda_{t} \Lambda_{y} \mathrm{Q}, \\
G_{\text {out }}(\lambda, w, \epsilon) & =h_{\text {out }}+h_{\text {in }}+\frac{1}{\lambda^{2}}\left(1-\chi_{\text {in }}\right) V(y)\left(\Theta \chi_{\text {out }}+w\right)+\frac{\lambda_{t}}{\lambda^{\frac{n}{2}}}\left(1-\chi_{\text {in }}\right) \Lambda_{y} \mathrm{Q}(y)+\mathrm{N}(v) .
\end{aligned}
$$

We can check that $v(x, t)$ defied in (5.3) gives a solution of (5.1), if $(\epsilon(y, t), w(x, t))$ solves (5.5). By a lack of boundary condition in the equation for $\epsilon(y, t)$ in $(5.5)$, the problem may not be uniquely solvable. So we appropriately construct a solution $\epsilon(y, t)$ such that $\epsilon(y, t)$ decays enough in the region $|y| \sim R$ (see 6.16$)$.

\subsection{Fixed point argument}

To construct a solution of (5.5), we apply a fixed point argument. We put

$$
\mathcal{W}(x, t)=\left\{\begin{array}{ll}
(T-t)^{l}\left(1+|z|^{2 l+2}\right) & \text { for }|z|<(T-t)^{-\frac{l}{2 l+2}}, \\
\frac{1}{1+|x|^{2}} & \text { for }|z|>(T-t)^{-\frac{l}{2 l+2}},
\end{array} \quad z=\frac{x}{\sqrt{T-t}} .\right.
$$

We fix two small positive constants $\delta_{0}$ and $\sigma$. Let $X_{\sigma}$ be the space of all continuous functions on $\mathbb{R}^{5} \times[0, T-2 \sigma]$ satisfying

$$
|w(x, t)| \leq \delta_{0} \mathcal{W}(x, t) \quad \text { for } t \in[0, T-2 \sigma]
$$

The metric in $X_{\sigma}$ is defined by

$$
d_{X_{\sigma}}\left(w_{1}, w_{2}\right)=\left\|w_{1}-w_{2}\right\|_{C\left(\mathbb{R}^{5} \times[0, T-2 \sigma]\right)} .
$$

We extend $w(x, t)$ to a continuous function on $\mathbb{R}^{5} \times[0, T]$.

$$
\bar{w}(x, t)= \begin{cases}w(x, t) & \text { if }(x, t) \in \mathbb{R}^{5} \times(0, T-2 \sigma), \\ \chi_{\sigma}(t) w(x, T-2 \sigma) & \text { if }(x, t) \in \mathbb{R}^{5} \times(T-2 \sigma, T),\end{cases}
$$

where $\chi_{\sigma}(t)$ is a cut off function satisfying $\chi_{\sigma}(t)=1$ if $t \in\left[0, T-\frac{3}{2} \sigma\right]$ and $\chi_{\sigma}(t)=0$ if $t \in[T-\sigma, T]$. Furthermore we define

$$
\tilde{w}(x, t)= \begin{cases}\bar{w}(x, t) & \text { if } t \in[0, T-2 \sigma], \\ \delta_{0} \mathcal{W}(x, t) & \text { if } t \in[T-2 \sigma, T] \text { and } \bar{w}(x, t)>\delta_{0} \mathcal{W}(x, t), \\ \bar{w}(x, t) & \text { if } t \in[T-2 \sigma, T] \text { and }|\bar{w}(x, t)| \leq \delta_{0} \mathcal{W}(x, t), \\ -\delta_{0} \mathcal{W}(x, t) & \text { if } t \in[T-2 \sigma, T] \text { and } \bar{w}(x, t)<-\delta_{0} \mathcal{W}(x, t) .\end{cases}
$$

From this definition, we see that $\tilde{w}(x, t) \in C\left(\mathbb{R}^{5} \times[0, T]\right)$ and

$$
|\tilde{w}(x, t)| \leq \begin{cases}\delta_{0} \mathcal{W}(x, t) & \text { for }(x, t) \in \mathbb{R}^{5} \times(0, T-\sigma) \\ 0 & \text { for }(x, t) \in \mathbb{R}^{5} \times(T-\sigma, T) .\end{cases}
$$


For given $w(x, t) \in X_{\sigma}$, we first determine $\lambda(t)$ by the orthogonal condition (6.1). Next we construct $\epsilon(y, t)$ as a solution of

$$
\lambda^{2} \epsilon_{t}=H_{y} \epsilon+G_{\text {in }}(\lambda, \tilde{w}) \quad \text { in } B_{2 R} \times(0, T) .
$$

After that we solve the problem

$$
W_{t}=\Delta_{x} W+G_{\text {out }}(\lambda, \tilde{w}, \epsilon) \quad \text { in } \mathbb{R}^{5} \times(0, T) .
$$

By using this $W(x, t)$, we define the mapping $w(x, t) \mapsto W(x, t)$. The fixed point of this mapping gives the desired solution of 5.5. Finally we take $\sigma \rightarrow 0$ to obtain the solution described in Theorem 1. For the rest of paper, we construct the solution mapping in the above procedure.

\subsection{Notations}

From now on, we assume $R \gg 1$ and choose $T$ as

$$
T=e^{-R}
$$

For any positive constant $k_{1}$ and $k_{2}$, we write

$$
k_{1} \lesssim k_{2}
$$

if there is a universal constant $c>0$ independent of $R, \delta_{0}, \sigma$ such that $k_{1} \leq c k_{2}$.

\section{Inner solution}

In this section, we repeat the argument in Lemma 4.1 [ 6] to define the mapping $w(x, t) \in X_{\sigma} \mapsto \epsilon(y, t)$ mentioned in Section 5.2. Throughout this section, $\tilde{w}(x, t) \in C\left(\mathbb{R}^{5} \times[0, T]\right)$ represents an extension of $w(x, t) \in X_{\sigma}$ defined in Section 5.2

\subsection{Choice of $\lambda(t)$}

We define $\lambda(t) \in C^{1}([0, T])$ as the unique solution of

$$
\left(\chi_{4 R} G_{\text {in }}(t), \Lambda_{y} \mathrm{Q}\right)_{L_{y}^{2}\left(B_{8 R}\right)}=0 \quad \text { for } t \in(0, T) \quad \text { and } \quad \lambda(T)=0,
$$

where $G_{\text {in }}(t)=G_{\text {in }}(\lambda(t), \tilde{w}(\lambda(t) y, t))$ and $\chi_{4 R}(y)=\chi\left(\frac{|y|}{4 R}\right)$.

Lemma 6.1. There exists $K>1$ independent of $R, \delta_{0}, \sigma$ such that

$$
\begin{aligned}
\left|\lambda(t)-\alpha_{l}^{2}(T-t)^{2 l+2}\right| & <K\left(\delta_{0}+\frac{T-t}{R^{2}}\right)(T-t)^{2 l+2} & & \text { for } t \in(0, T), \\
\left|\frac{d \lambda}{d t}(t)-(2 l+2) \alpha_{l}^{2}(T-t)^{2 l+1}\right| & <K\left(\delta_{0}+\frac{T-t}{R^{2}}\right)(T-t)^{2 l+1} & & \text { for } t \in(0, T),
\end{aligned}
$$

where $\alpha_{l}=-\frac{\left(\chi_{4 R} V, \Lambda_{y} Q\right)_{L_{y}^{2}\left(B_{8 R}\right)}}{2(l+1)\left(\chi_{4 R} \Lambda_{y} Q, \Lambda_{y} Q\right)_{L 2}^{2}\left(B_{8 R}\right)}$.

Proof. From (5.6), the orthogonal condition (6.1) is explicitly given by

$$
\sqrt{\lambda}\left(\chi_{4 R} V \Theta(t), \Lambda_{y} \mathrm{Q}\right)_{L_{y}^{2}\left(B_{8 R}\right)}+\sqrt{\lambda}\left(\chi_{4 R} V \tilde{w}(t), \Lambda_{y} \mathrm{Q}\right)_{L_{y}^{2}\left(B_{8 R}\right)}+\frac{d \lambda}{d t}\left(\chi_{4 R} \Lambda_{y} \mathrm{Q}, \Lambda_{y} \mathrm{Q}\right)_{L_{y}^{2}\left(B_{8 R}\right)}=0 .
$$

Put $C(t)=\sqrt{\lambda(t)}$. The function $C(t)$ satisfies

$$
\frac{d C}{d t}=-\frac{\left(\chi_{4 R} V \Theta(x, t), \Lambda_{y} \mathrm{Q}\right)_{L_{y}^{2}\left(B_{8 R}\right)}+\left(\chi_{4 R} V \tilde{w}(x, t), \Lambda_{y} \mathrm{Q}\right)_{L_{y}^{2}\left(B_{8 R}\right)}}{2\left(\chi_{4 R} \Lambda_{y} \mathrm{Q}, \Lambda_{y} \mathrm{Q}\right)_{L_{y}^{2}\left(B_{8 R}\right)}}, \quad x=C^{2} y .
$$

We now show the unique solvability of the problem 6.2 in

$$
\mathcal{S}=\left\{C(t) \in C([0, T]) ; 0 \leq C(t) \leq \frac{\sqrt{T-t}}{R}\right\} .
$$

Let us consider

$$
\frac{d D}{d t}=-\frac{\left(\chi_{4 R} V \Theta(x, t), \Lambda_{y} \mathrm{Q}\right)_{L_{y}^{2}\left(B_{8 R}\right)}+\left(\chi_{4 R} V \tilde{w}(x, t), \Lambda_{y} \mathrm{Q}\right)_{L_{y}^{2}\left(B_{8 R}\right)}}{2\left(\chi_{4 R} \Lambda_{y} \mathrm{Q}, \Lambda_{y} \mathrm{Q}\right)_{L_{y}^{2}\left(B_{8 R}\right)}}, \quad x=C^{2} y .
$$


This differential equation defines the mapping $C(t) \mapsto D(t)$. Put $z=\frac{x}{\sqrt{T-t}}$. Since $e_{l}(z)=1+a_{1}|z|^{2}+a_{2}|z|^{4}+\cdots+a_{l}|z|^{2 l}$, the first term on the right-hand side is written as

$$
\begin{aligned}
\left(\chi_{4 R} V \Theta(x, t), \Lambda_{y} \mathrm{Q}\right)_{L_{y}^{2}\left(B_{8 R}\right)} & =-(T-t)^{l}\left(V e_{l}(z), \Lambda_{y} \mathrm{Q}\right)_{L_{y}^{2}\left(B_{8 R}\right)} \\
& =-(T-t)^{l}\left(\left(\chi_{4 R} V, \Lambda_{y} \mathrm{Q}\right)_{L_{y}^{2}\left(B_{8 R}\right)}+\sum_{k=1}^{l} a_{k}\left(\chi_{4 R} V|z|^{2 k}, \Lambda_{y} \mathrm{Q}\right)_{L_{y}^{2}\left(B_{8 R}\right)}\right) \\
& =-(T-t)^{l}(\underbrace{\left(\chi_{4 R} V, \Lambda_{y} \mathrm{Q}\right)_{L_{y}^{2}\left(B_{8 R}\right)}}_{=: b_{0}}+\sum_{k=1}^{l} \underbrace{a_{k}\left(\chi_{4 R} V\left(\frac{|y|}{R}\right)^{2 k}, \Lambda_{y} \mathrm{Q}\right)_{L_{y}^{2}\left(B_{8 R}\right)}}_{=: b_{k}} \frac{R^{2 k} C(t)^{4 k}}{(T-t)^{k}}) .
\end{aligned}
$$

Since $\left|b_{k}\right| \lesssim 1$, we easily see that if $C(t) \in \mathcal{S}$

$$
\left|\sum_{k=1}^{l} b_{k} \frac{R^{2 k} C(t)^{4 k}}{(T-t)^{k}}\right| \lesssim \frac{T-t}{R^{2}} \quad \text { for } t \in(0, T) .
$$

Furthermore when $|z|=\frac{C(t)^{2}}{\sqrt{T-t}}|y|$ and $C(t) \in \mathcal{S}$, it holds that $|z|<1$ for $y \in B_{8 R}$. Therefore since $\tilde{w}(x, t)$ satisfies $(5.9)$, it follows that if $C(t) \in \mathcal{S}$

$$
\left|\left(\chi_{4 R} V \tilde{w}\left(C^{2} y, t\right), \Lambda_{y} \mathrm{Q}\right)_{L_{y}^{2}\left(B_{8 R}\right)}\right| \lesssim \delta_{0}(T-t)^{l}\left|\left(\chi_{4 R} V, \Lambda_{y} \mathrm{Q}\right)_{L_{y}^{2}\left(B_{8 R}\right)}\right| \lesssim \delta_{0}(T-t)^{l} .
$$

Therefore since $b_{0}<0$ (see 4.3 ), we get if $C(t) \in \mathcal{S}$

$$
\frac{d D}{d t}=-\left(1+O\left(\delta_{0}\right)+O\left(\frac{T-t}{R^{2}}\right)\right) \underbrace{\left(\frac{\left|b_{0}\right|}{2\left(\chi_{4 R} \Lambda_{y} \mathrm{Q}, \Lambda_{y} \mathrm{Q}\right)_{L_{y}^{2}\left(B_{8 R}\right)}}\right)}_{=:(l+1) \alpha_{l}}(T-t)^{l} .
$$

This implies

$$
D(t)=\left(1+O\left(\delta_{0}\right)+O\left(\frac{T-t}{R^{2}}\right)\right) \alpha_{l}(T-t)^{l+1} .
$$

Therefore we proved that $D(t) \in \mathcal{S}$ if $C(t) \in \mathcal{S}$. As a consequence, by a fixed point argument, we obtain a solution $C(t)$ of 6.2 satisfying (6.3). Next we prove the uniqueness for solutions of $(6.2)$ in $\mathcal{S}$. Let $C_{1}(t), C_{2}(t) \in \mathcal{S}$ be two solutions of 6.2. Since $\tilde{w}(x, t)=0$ for $t \in(T-\sigma, T)$, it is clear that $C_{1}(t)=C_{2}(t)$ for $t \in(T-\sigma, T)$. We write $x_{1}=C_{1}^{2} y$, $x_{2}=C_{2}^{2} y$ and $y^{\prime}=\frac{C_{1}^{2}}{C_{2}^{2}} y$. By the change of variables, we see that

$$
\begin{aligned}
\left|\frac{d}{d t}\left(C_{1}-C_{2}\right)\right| \lesssim\left|\int_{B_{8 R}} \chi_{4 R} V \tilde{w}\left(x_{1}, t\right) \Lambda_{y} \mathrm{Q} d y-\int_{B_{8 R}} \chi_{4 R} V \tilde{w}\left(x_{2}, t\right) \Lambda_{y} \mathrm{Q} d y\right| \\
=\left|\int_{B_{8 R}} \chi_{4 R} V \tilde{w}\left(x_{1}, t\right) \Lambda_{y} \mathrm{Q} d y-\left(\frac{C_{1}}{C_{2}}\right)^{10} \int_{B_{8\left(\frac{C_{2}}{C_{1}}\right)^{2} R}} \chi_{4 R}\left(y^{\prime}\right) V\left(y^{\prime}\right) \tilde{w}\left(x_{1}, t\right) \Lambda_{y} \mathrm{Q}\left(y^{\prime}\right) d y\right| \\
\lesssim\left|1-\left(\frac{C_{1}}{C_{2}}\right)^{10}\right| \int_{B_{8 R}}\left|\chi_{4 R} V \tilde{w}\left(x_{1}, t\right) \Lambda_{y} \mathrm{Q}\right| d y+\left(\frac{C_{1}}{C_{2}}\right)^{10} \mid \int_{B_{8 R}} \chi_{4 R} V \tilde{w}\left(x_{1}, t\right) \Lambda_{y} \mathrm{Q} d y \\
\quad-\int_{B_{8\left(\frac{C_{2}}{C_{1}}\right)^{2} R} \chi_{4 R}\left(y^{\prime}\right) V\left(y^{\prime}\right) \tilde{w}(x, t) \Lambda_{y} \mathrm{Q}\left(y^{\prime}\right) d y \mid}
\end{aligned}
$$

Repeating the above argument, we can verify that $C_{1}(t), C_{2}(t)$ satisfy 6.3$)$. Therefore we find that $C_{1}(t), C_{2}(t)>\frac{\alpha_{l}}{2} \sigma^{l+1}$ for $t \in(0, T-\sigma)$. As a consequence, there exists $c_{\sigma}>0$ such that

$$
\left|\frac{d}{d t}\left(C_{1}-C_{2}\right)\right|<c_{\sigma}\left|C_{1}-C_{2}\right| \quad \text { for } t \in(0, T-\sigma) .
$$

This assures the uniqueness of solutions in $t \in(0, T-\sigma)$. Therefore the uniqueness of 6.2 in $\mathcal{S}$ is proved. Since $C(t)=\sqrt{\lambda(t)}$, we obtain the conclusion. 


\subsection{Construction of $\epsilon(y, t)$}

Throughout this subsection, $\lambda(t)$ represents the function given in Lemma 6.1. For simplicity, we write

$$
G_{\text {in }}(t)=G_{\text {in }}(\lambda(t), \tilde{w}(\lambda(t) y, t)) .
$$

We define a function $g(y, t) \in C\left(\mathbb{R}^{5} \times[0, T]\right)$ as a solution of

$$
\begin{cases}-H_{y} g=\chi_{4 R} G_{\text {in }}(t) & \text { in } B_{8 R} \\ g=0 & \text { on } \partial B_{8 R} \\ g(\cdot, t) \text { is radial. } & \end{cases}
$$

Since $\left(\chi_{4 R} G_{\mathrm{in}}(t), \Lambda_{y} \mathrm{Q}\right)_{L_{y}^{2}\left(B_{8 R}\right)}=0$ for $t \in(0, T)$ (see 6.1$)$ ), the radial solution $g(r, t)$ is given by

$$
g(r, t)=k \Gamma(r) \int_{r}^{8 R} \Lambda_{y} \mathrm{Q} \chi_{4 R} G_{\text {in }}(t) r^{\prime 4} d r^{\prime}-k \Lambda_{y} \mathrm{Q}(r) \int_{r}^{8 R} \Gamma \chi_{4 R} G_{\text {in }}(t) r^{\prime 4} d r^{\prime}
$$

for some constant $k \in \mathbb{R}$ depending on $\Lambda_{y} \mathrm{Q}(r)$ and $\Gamma(r)$. The function $\Gamma(r)$ is a radial solution of $H_{y} \psi=0$ given in the proof of Lemma 3.3 . From (5.6) and Lemma 6.1, we verify that

$$
\begin{aligned}
\left|G_{\text {in }}\right| & =\left|\lambda^{\frac{3}{2}} \Theta(x, t) V+\lambda^{\frac{3}{2}} \tilde{w}(x, t) V+\lambda \lambda_{t} \Lambda_{y} \mathrm{Q}\right| \\
& \lesssim \frac{\lambda^{\frac{3}{2}}(T-t)^{l}}{1+|y|^{4}}+\frac{\lambda\left|\lambda_{t}\right|}{1+|y|^{3}}<\frac{\lambda^{\frac{3}{2}} \lambda^{\frac{l}{2 l+2}}}{1+|y|^{4}}+\frac{\lambda \lambda^{\frac{2 l+1}{2 l+2}}}{1+|y|^{3}} \\
& \lesssim \frac{\lambda^{\frac{4 l+3}{2 l+2}}}{1+|y|^{3}} \quad \text { for }|y|<8 R .
\end{aligned}
$$

Therefore by a direct computation, we get

$$
|g(y, t)|<\frac{\lambda^{\frac{4 l+3}{2 l+2}}}{1+|y|} \quad \text { for }|y|<8 R .
$$

For simplicity, we put

$$
\gamma=\frac{4 l+3}{2 l+2}
$$

We introduce a new time variable $s$ defined by

$$
\frac{d s}{d t}=\frac{1}{\lambda(t)^{2}} \quad \text { and }\left.\quad s(t)\right|_{t=0}=0 .
$$

Since $\frac{\alpha_{l}^{2}}{2}(T-t)^{2 l+2}<\lambda<2 \alpha_{l}^{2}(T-t)^{2 l+2}$ (see Lemma 6.1), it is expressed in the variable $s$.

$$
\frac{\alpha_{l}^{2}}{2}\left(\frac{1}{T^{-(4 l+3)}+\frac{4 l+3}{4} \alpha_{l}^{2} s}\right)^{\frac{2 l+2}{4 l+3}}<\lambda<2 \alpha_{l}^{2}\left(\frac{1}{T^{-(4 l+3)}+4(4 l+3) \alpha_{l}^{2} s}\right)^{\frac{2 l+2}{4 l+3}} .
$$

Let $\mu_{1}^{(8 R)}<0$ and $\psi_{1}^{(8 R)}(y) \in H_{0}^{1}\left(B_{8 R}\right)$ be defined in Section 3.2 . We consider

$$
\begin{cases}\partial_{s} E=H_{y} E+g(y, s) & \text { in } B_{8 R} \times(0, \infty), \\ E=0 & \text { on } \partial B_{8 R} \times(0, \infty), \\ E=\frac{\mathrm{d}_{\text {in }}}{\mu_{1}^{(8 R)}} \psi_{1}^{(8 R)} & \text { for } s=0 .\end{cases}
$$

The parameter $\mathrm{d}_{\mathrm{in}}$ is determined below. The desired solution $\epsilon(y, t)$ mentioned in section 5.2 is obtained by $\epsilon(y, t)=$ $H_{y} E(y, t)$. Let $M_{1}$ be the constant given in Lemma 3.4 and fix a large constant $M>M_{1}$ such that

$$
|y|^{2} V(y)+|y|^{\frac{5}{2}} e^{-\sqrt{\left|\mu_{1}\right|}|y|} \ll 1 \quad \text { for }|y|>2 M .
$$

We first consider

$$
\begin{cases}\partial_{s} E_{1}=\Delta_{y} E_{1}+\left(1-\chi_{M}\right) V(y) E_{1}+g(y, s) & \text { in } B_{8 R} \times(0, \infty), \\ E_{1}=0 & \text { on } \partial B_{8 R} \times(0, \infty), \\ E_{1}=0 & \text { for } s=0 .\end{cases}
$$

Since $g(y, t)=0$ near $\partial B_{8 R} \times[0, \infty)$, by a certain approximation procedure, we can verify that there exists $\alpha \in(0,1)$ such that

$$
E_{1}, \nabla_{y} E_{1}, \Delta_{y} E_{1}, \nabla_{y} \Delta_{y} E_{1} \in C^{\alpha}\left(\bar{B}_{8 R} \times[0, \infty)\right) .
$$


Lemma 6.2. There exists $K_{1}>1$ independent of $R, \delta_{0}, \sigma$ such that

$$
\left|E_{1}(y, s)\right|+\left|\nabla E_{1}(y, s)\right| \leq K_{1} R \lambda^{\gamma} \quad \text { for }(y, s) \in B_{8 R} \times(0, \infty) .
$$

Proof. To construct a comparison function, we put

$$
p(r)=p_{1}(r) \int_{r}^{16 R} \frac{d r_{1}}{p_{1}\left(r_{1}\right)^{2} r_{1}^{n-1}} \int_{0}^{r_{1}} \frac{p_{1}\left(r_{2}\right) r_{2}^{n-1}}{1+r_{2}} d r_{2},
$$

where $p_{1}(r)$ is a radial function given in Lemma 3.4. The function $p(r)$ gives a positive radial solution of

$$
\begin{cases}\Delta_{y} p+\left(1-\chi_{M}\right) V p+\frac{1}{1+|y|}=0 & \text { in } B_{16 R} \\ p=0 & \text { on } \partial B_{16 R}\end{cases}
$$

Since $k<p_{1}(r)<1$ for $r>0$ (see Lemma 3.4 ), there exist $k_{1}, k_{2}>0$ independent of $M, R$ such that

$$
k_{1} R<p(r)<k_{2} R \quad \text { for } r \in(0,8 R)
$$

We now check that $K \lambda^{\gamma} p(y)$ gives a super-solution of 6.8 . Since $\left|\lambda \frac{d \lambda}{d t}\right|<(4 l+4) \alpha_{l}^{4}(T-t)^{4 l+3}<(4 l+4) \alpha_{l}^{4} T^{4 l+3}($ see Lemma 6.1, we see from 6.5 and 6.10 that

$$
\begin{aligned}
\left(\partial_{s}-\Delta_{y}-\left(1-\chi_{M}\right) V\right) \lambda^{\gamma} p(y) & =\left(\frac{\gamma}{\lambda} \frac{d t}{d s} \frac{d \lambda}{d t} p(y)+\frac{1}{1+|y|}\right) \lambda^{\gamma} \\
& >\left(-\gamma \lambda \frac{d \lambda}{d t} p(y)+\frac{1}{1+|y|}\right) \lambda^{\gamma} \\
& >\left(-(4 l+4) \gamma \alpha_{l}^{4} k_{2} T^{4 l+3} R+\frac{1}{1+|y|}\right) \lambda^{\gamma}
\end{aligned}
$$

Since $T=e^{-R}$, it holds that

$$
(4 l+4) \gamma \alpha_{l}^{4} k_{2} T^{4 l+3} R<\frac{\frac{1}{2}}{1+8 R}<\frac{\frac{1}{2}}{1+|y|} \quad \text { for } y \in B_{8 R} .
$$

Therefore we obtain

$$
\left(\partial_{s}-\Delta_{y}-\left(1-\chi_{M}\right) V\right) K \lambda^{\gamma} p(y)>\frac{K}{2} \frac{\lambda^{\gamma}}{1+|y|} \quad \text { for } y \in B_{8 R} .
$$

Since $|g(y, s)| \lesssim \frac{\lambda^{\gamma}}{1+|y|}($ see 6.4$)$, by a comparison argument, we obtain if $K \gg 1$

$$
\left|E_{1}(y, s)\right|<K \lambda^{\gamma} p(y)<K k_{2} R \lambda^{\gamma} \quad \text { for }(y, s) \in B_{8 R} \times(0, \infty) .
$$

Applying a local parabolic estimate in 6.8, we get from 6.4 that

$$
\begin{aligned}
\left|\nabla_{y} E_{1}(y, s)\right| & \lesssim \sup _{\min \{s-1,0\}<s^{\prime}<s} \sup _{\left|y^{\prime}-y\right|<1}\left(\left|E_{1}\left(y^{\prime}, s^{\prime}\right)\right|+\left|g\left(y^{\prime}, s^{\prime}\right)\right|\right) \\
& \lesssim \sup _{\min \{s-1,0\}<s^{\prime}<s}\left(R \lambda\left(s^{\prime}\right)^{\gamma}+\frac{\lambda\left(s^{\prime}\right)^{\gamma}}{1+|y|}\right)
\end{aligned}
$$

From 6.6), we can verify that

$$
\sup _{\min \{s-1,0\}<s^{\prime}<s} \lambda\left(s^{\prime}\right) \lesssim \lambda(s)
$$

Therefore we complete the proof.

Lemma 6.3. There exists $K_{2}>1$ independent of $R, \delta_{0}, \sigma$ such that

$$
\left|\Delta_{y} E_{1}(y, s)\right|+|y| \cdot\left|\nabla_{y} \Delta_{y} E_{1}(y, s)\right|<K_{2}\left(\frac{\lambda^{\gamma}}{1+|y|}+\frac{R \lambda^{\gamma}}{1+|y|^{2}}\right) \quad \text { for }(y, s) \in B_{6 R} \times(0, \infty) .
$$


Proof. We put

$$
e_{1}=\Delta_{y} E_{1}+\left(1-\chi_{M}\right) V(y) E_{1} .
$$

We easily see from 6.8 - 6.9 that $e_{1}(y, s)$ solves

$$
\begin{cases}\partial_{s} e_{1}=\Delta_{y} e_{1}+\left(1-\chi_{M}\right) V(y) e_{1}+\left(\Delta_{y}+\left(1-\chi_{M}\right) V(y)\right) g(y, s) & \text { in } B_{8 R} \times(0, \infty), \\ e_{1}=0 & \text { on } \partial B_{8 R} \times(0, \infty), \\ e_{1}=0 & \text { for } s=0 .\end{cases}
$$

Since $\left|\left(\Delta_{y}+\left(1-\chi_{M}\right) V(y)\right) g(y, s)\right|<\frac{\lambda^{\gamma}}{1+|y|^{3}}$, by the same argument as in the proof of Lemma 6.2 we verify that

$$
e_{1}(y, s) \lesssim \frac{\lambda^{\gamma}}{1+|y|} \quad \text { for }(y, s) \in B_{8 R} \times(0, \infty)
$$

To extend $e_{1}(y, s)$ to $s<0$, we put

$$
\bar{e}_{1}(y, s)=\left\{\begin{array}{ll}
e_{1}(y, s) & \text { if } s \geq 0, \\
0 & \text { if } s<0,
\end{array} \quad \bar{g}(y, s)= \begin{cases}g(y, s) & \text { if } s \geq 0 \\
0 & \text { if } s<0\end{cases}\right.
$$

Since $e_{1}, \nabla_{y} e_{1} \in C^{\alpha}\left(\bar{B}_{8 R} \times[0, \infty)\right)($ see 6.9$)$, we find that $\bar{e}_{1}, \nabla_{x} \bar{e}_{1} \in C^{\alpha}\left(\bar{B}_{8 R} \times(-\infty, \infty)\right)$ and $\bar{e}_{1}(y, s)$ solves

$$
\begin{cases}\partial_{s} \bar{e}_{1}=\Delta_{y} \bar{e}_{1}+\left(1-\chi_{M}\right) V(y) \bar{e}_{1}+\left(\Delta_{y}+\left(1-\chi_{M}\right) V(y)\right) \bar{g}(y, s) & \text { in } B_{8 R} \times(-\infty, \infty), \\ \bar{e}_{1}=0 & \text { on } \partial B_{8 R} \times(-\infty, \infty) .\end{cases}
$$

We fix $(y, s) \in B_{6 R} \backslash B_{1} \times(0, \infty)$. We write $\rho=|y|$ and define

$$
\tilde{e}_{1}(Y, S)=\bar{e}_{1}\left(y+\frac{\rho}{3} Y, s+\frac{\rho^{2}}{9}(S-1)\right) .
$$

We easily see that $\tilde{e}_{1}(Y, S)$ solves

$$
\partial_{S} \tilde{e}_{1}=\Delta_{Y} \tilde{e}_{1}+\frac{\rho^{2}}{9}\left(1-\chi_{M}\right) V \tilde{e}_{1}+\frac{\rho^{2}}{9}\left(\Delta_{y}+2\left(1-\chi_{M}\right) V\right) \bar{g} \quad \text { in } B_{1} \times(0,1) .
$$

Since $|y|=\rho$, it holds that

$$
\frac{\rho^{2}}{9}\left(1-\chi_{M}\right) V \lesssim \frac{\rho^{2}}{1+\left|y+\frac{\rho}{2} Y\right|^{4}} \lesssim \frac{\rho^{2}}{1+\rho^{4}} \quad \text { for }|Y|<1 .
$$

Therefore we get from Lemma 3.5 that

$$
\begin{aligned}
\left\|\tilde{e}_{1}\right\|_{C_{(Y, S)}^{\alpha}\left(B_{\frac{1}{2}} \times\left(\frac{1}{2}, 1\right)\right)} & +\left\|\nabla_{Y} \tilde{e}_{1}\right\|_{C_{(Y, S)}^{\alpha}\left(B_{\frac{1}{2}} \times\left(\frac{1}{2}, 1\right)\right)} \\
& \lesssim\left\|\tilde{e}_{1}\right\|_{L_{(Y, S)}^{\infty}\left(B_{1} \times(0,1)\right)}+\left\|\frac{\rho^{2}}{9}\left(\Delta_{y}+2\left(1-\chi_{M}\right) V\right) \bar{g}\right\|_{L_{(Y, S)}^{\infty}\left(B_{1} \times(0,1)\right)} .
\end{aligned}
$$

Since $\left|\left(\Delta_{y}+\left(1-\chi_{M}\right) V(y)\right) g(y, s)\right|<\frac{\lambda^{\gamma}}{1+|y|^{3}}$, we see that

$$
\left\|\frac{\rho^{2}}{9}\left(\Delta_{y}+2\left(1-\chi_{M}\right) V\right) \bar{g}\right\|_{L_{(Y, S)}^{\infty}\left(B_{1} \times(0,1)\right)} \lesssim \frac{\rho^{2}}{1+\rho^{3}}\left(\sup _{\min \left\{0, s-\frac{\rho^{2}}{9}\right\}<s^{\prime}<s} \lambda\left(s^{\prime}\right)\right)^{\gamma} .
$$

From 6.6 and $T=e^{-R}$, we verify that

$$
\sup _{\min \left\{0, s-\frac{\rho^{2}}{9}\right\}<s^{\prime}<s} \lambda\left(s^{\prime}\right) \lesssim \lambda(s) .
$$

Since $\nabla_{Y} \tilde{e}_{1}(Y, S)=\frac{\rho}{3} \nabla_{y} \bar{e}_{1}\left(y+\frac{\rho}{3} Y, s+\frac{\rho^{2}}{9} S\right)$, we deduce from 6.12 that

$$
\begin{aligned}
\frac{\rho}{3}\left|\nabla_{y} e_{1}(y, s)\right| & <\left\|\nabla_{Y} \tilde{e}_{1}\right\|_{C_{(Y, S)}^{\alpha}\left(B_{\frac{1}{2}} \times\left(\frac{1}{2}, 1\right)\right)} \\
& \lesssim\left\|\tilde{e}_{1}\right\|_{L_{(Y, S)}^{\infty}\left(B_{1} \times(0,1)\right)}+\frac{\rho^{2}}{1+\rho^{3}} \lambda(s)^{\gamma} \\
& \lesssim \frac{\lambda^{\gamma}}{1+\rho}
\end{aligned}
$$


Therefore it follows that

$$
\left|\nabla_{y} e_{1}(y, s)\right| \lesssim \frac{\lambda^{\gamma}}{1+|y|^{2}} \quad \text { for }(y, s) \in B_{6 R} \backslash B_{1} \times(0, \infty) .
$$

Combining this estimate and 6.12 , we obtain

$$
\left|\nabla_{y} e_{1}(y, s)\right| \lesssim \frac{\lambda^{\gamma}}{1+|y|^{2}} \quad \text { for }(y, s) \in B_{6 R} \times(0, \infty)
$$

From definition of $e_{1}(y, s)$ and Lemma 6.2 we complete the proof.

Next we put

$$
E_{2}=E-E_{1}
$$

The function $E_{2}(y, s)$ solves

$$
\begin{cases}\partial_{s} E_{2}=H_{y} E_{2}+\chi_{M} V(y) E_{1} & \text { in } B_{8 R} \times(0, \infty) \\ E_{2}=0 & \text { on } \partial B_{8 R} \times(0, \infty) \\ E_{2}=\frac{\mathrm{d}_{\text {in }}}{\mu_{1}^{(8 R)}} \psi_{1}^{(8 R)} & \text { for } s=0\end{cases}
$$

We now take

$$
\mathrm{d}_{\mathrm{in}}=-\mu_{1}^{(8 R)} \int_{0}^{\infty} e^{\mu_{1}^{(8 R)} s^{\prime}}\left(\chi_{M} V E_{1}\left(s^{\prime}\right), \psi_{1}^{(8 R)}\right)_{L_{y}^{2}\left(B_{8 R}\right)} d s^{\prime}
$$

and define $\mathrm{c}(s)$ by

$$
\left\{\begin{array}{l}
\frac{d \mathrm{c}}{d s}=-\mu_{1}^{(8 R)} \mathrm{c}+\left(\chi_{M} V E_{1}(s), \psi_{1}^{(8 R)}\right)_{L_{y}^{2}\left(B_{8 R}\right)} \\
\mathrm{c}(0)=\frac{\mathrm{d}_{\text {in }}}{\mu_{1}^{(8 R)}}
\end{array}\right.
$$

The function $\mathrm{c}(s)$ is explicitly given by

$$
\mathrm{c}(s)=-\int_{s}^{\infty} e^{-\mu_{1}^{(8 R)}\left(s-s^{\prime}\right)}\left(\chi_{M} V E_{1}\left(s^{\prime}\right), \psi_{1}^{(8 R)}\right)_{L_{y}^{2}\left(B_{8 R}\right)} d s^{\prime} .
$$

From 6.6 and Lemma 6.2 , we easily see that

$$
|\mathrm{c}(s)| \lesssim R \lambda^{\gamma},\left.\quad\left|\mathrm{d}_{\mathrm{in}}\right| \lesssim R \lambda(s)\right|_{s=0} ^{\gamma} \lesssim R T^{4 l+3} .
$$

We decompose $E_{2}(y, s)$ as

$$
E_{2}=\nu+\mathrm{c}(s) \psi_{1}^{(8 R)}
$$

The function $\nu(y, s)$ satisfies

$$
\begin{cases}\partial_{s} \nu=H_{y} \nu+V \chi_{M} E_{1}-\left(\chi_{M} V E_{1}, \psi_{1}^{(8 R)}\right)_{L_{y}^{2}\left(B_{8 R}\right)} \psi_{1}^{(8 R)} & \text { in } B_{8 R} \times(0, \infty) \\ \nu=0 & \text { on } \partial B_{8 R} \times(0, \infty) \\ \nu=0 & \text { for } s=0 .\end{cases}
$$

Lemma 6.4. There exists $K_{3}>1$ independent of $R, \delta_{0}, \sigma$ such that

$$
|\nu(y, s)|+|y| \cdot\left|\nabla_{y} \nu(y, s)\right|+|y|^{2} \cdot\left|\Delta_{y} \nu(y, s)\right|+|y|^{3} \cdot\left|\nabla_{y} \Delta_{y} \nu(y, s)\right|<\frac{K_{3} R^{4} \lambda^{\gamma}}{1+|y|^{\frac{5}{2}}}
$$

for $(y, s) \in B_{2 R} \times(0, \infty)$.

Proof. Since $\left(\nu(s), \psi_{1}^{(8 R)}\right)_{L_{y}^{2}\left(B_{8 R}\right)}=0$ for $s \in(0, \infty)$, from Lemma 3.3 there exists $k>0$ such that

$$
\left(H_{y} \nu(s), \nu(s)\right)_{L_{y}^{2}\left(B_{8 R}\right)}<-\frac{k}{R^{3}}\|\nu(s)\|_{L_{y}^{2}\left(B_{8 R}\right)} \quad \text { for } s \in(0, \infty) .
$$

From this estimate and Lemma 6.2, we get

$$
e^{\frac{k}{R^{3}} s}\|\nu\|_{L_{y}^{2}\left(B_{8 R}\right)}^{2} \lesssim R^{5}\left\|\chi_{M} V\right\|_{L_{y}^{2}\left(B_{8 R}\right)}^{2} \int_{0}^{s} e^{\frac{k}{R^{3}} s^{\prime}} \lambda\left(s^{\prime}\right)^{2 \gamma} d s^{\prime} .
$$


We calculate the integral using Lemma 6.1 and 6.5).

$$
\begin{aligned}
\int_{0}^{s} e^{\frac{k}{R^{3}} s^{\prime}} \lambda\left(s^{\prime}\right)^{2 \gamma} d s^{\prime} & <R^{3} e^{\frac{k}{R^{3}} s} \lambda(s)^{2 \gamma}-2 \gamma R^{3} \int_{0}^{s} e^{\frac{k}{R^{3}} s^{\prime}} \lambda^{2 \gamma-1} \frac{d \lambda}{d s} d s^{\prime} \\
& =R^{3} e^{\frac{k}{R^{3}} s} \lambda(s)^{2 \gamma}-2 \gamma R^{3} \int_{0}^{s} e^{\frac{k}{R^{3}} s^{\prime}} \lambda^{2 \gamma+1} \frac{d \lambda}{d t} d s^{\prime} \\
& \lesssim R^{3} e^{\frac{k}{R^{3}} s} \lambda(s)^{2 \gamma}+R^{3} \int_{0}^{s} e^{\frac{k}{R^{3}} s^{\prime}} \lambda^{2 \gamma+1} \lambda^{\frac{2 l+1}{2 l+2}} d s^{\prime} \\
& \lesssim R^{3} e^{\frac{k}{R^{3}} s} \lambda(s)^{2 \gamma}+\left.R^{3} \lambda(s)\right|_{s=0} ^{\frac{4 l+3}{2 l+2}} \int_{0}^{s} e^{\frac{k}{R^{3}} s^{\prime}} \lambda^{2 \gamma} d s^{\prime}
\end{aligned}
$$

Since $\left.\lambda(s)\right|_{s=0}=\left.\lambda(t)\right|_{t=0} \lesssim T^{2 l+2}$ and $T=e^{-R}$, it follows that $\left.R^{3} \lambda(s)\right|_{s=0} ^{\frac{4 l+3}{2 l+2}}<\frac{1}{2}$. Therefore we obtain

$$
\int_{0}^{s} e^{\frac{k}{R^{3}} s^{\prime}} \lambda\left(s^{\prime}\right)^{2 \gamma} d s^{\prime} \lesssim R^{3} e^{\frac{k}{R^{3}} s} \lambda(s)^{2 \gamma}
$$

As a consequence, we deduce that

$$
\|\nu(s)\|_{L_{y}^{2}\left(B_{8 R}\right)} \lesssim R^{4} \lambda^{\gamma} .
$$

Applying a local parabolic estimate in 6.14, we get from 6.11) that

$$
\|\nu(s)\|_{L_{y}^{\infty}\left(B_{8 R}\right)} \lesssim R^{4} \lambda^{\gamma} .
$$

We now check that $K R^{4} \lambda^{\gamma}|y|^{-\frac{5}{2}}$ becomes a super solution for $y \in B_{8 R} \backslash B_{2 M}$. From (6.7), Lemma 6.1 and 6.5, we see that

$$
\begin{aligned}
\left(\partial_{s}-H_{y}\right)\left(\frac{\lambda^{\gamma}}{|y|^{\frac{5}{2}}}\right) & =\left(\frac{\gamma}{\lambda} \frac{d \lambda}{d t} \frac{d t}{d s}+\frac{5}{4|y|^{2}}-V(y)\right)\left(\frac{\lambda^{\gamma}}{|y|^{\frac{5}{2}}}\right) \\
& >\left(\gamma \lambda \frac{d \lambda}{d t}+\frac{5}{8|y|^{2}}\right)\left(\frac{\lambda^{\gamma}}{|y|^{\frac{5}{2}}}\right) \\
& >\left(-(4 l+4) \gamma \alpha_{l}^{4} T^{4 l+3}+\frac{5}{8|y|^{2}}\right)\left(\frac{\lambda^{\gamma}}{|y|^{\frac{2}{2}}}\right)
\end{aligned}
$$

for $y \in B_{8 R} \backslash B_{2 M}$. Since $T=e^{-R}$, we note that $(4 l+4) \gamma \alpha_{l}^{4} T^{4 l+3}<\frac{5}{16|y|^{2}}$ for $y \in B_{8 R}$. Therefore we get

$$
\left(\partial_{s}-H_{y}\right)\left(\frac{K R^{4} \lambda^{\gamma}}{|y|^{\frac{5}{2}}}\right)>K R^{4}\left(\frac{5}{16|y|^{2}}\right)\left(\frac{\lambda^{\gamma}}{|y|^{\frac{5}{2}}}\right) \quad \text { for } y \in B_{8 R} \backslash B_{2 M}
$$

Furthermore from Lemma 3.1, 6.7 and Lemma 6.2 we see that

$$
\left(V \chi_{M} E_{1}, \psi_{1}^{(8 R)}\right)_{L_{y}^{2}\left(B_{8 R}\right)} \psi_{1}^{(8 R)}(y) \lesssim R \lambda^{\gamma} \frac{e^{-\sqrt{\left|\mu_{1}\right|} \cdot|y|}}{|y|^{2}} \lesssim \frac{R \lambda^{\gamma}}{|y|^{2+\frac{5}{2}}} \quad \text { for } y \in B_{8 R} \backslash B_{2 M} .
$$

Therefore it holds that if $K \gg 1$

$$
\left(\partial_{s}-H_{y}\right)\left(\frac{K R^{4} \lambda^{\gamma}}{|y|^{\frac{5}{2}}}\right)>\left(V \chi_{M} E_{1}, \psi_{1}^{(8 R)}\right)_{L_{y}^{2}\left(B_{8 R}\right)} \psi_{1}^{(8 R)} \quad \text { for } y \in B_{8 R} \backslash B_{2 M} .
$$

Combining this estimate and 6.15, by a comparison argument in 6.14, we obtain

$$
|\nu(y, s)|<\frac{K R^{4} \lambda^{\gamma}}{|y|^{\frac{5}{2}}} \quad \text { for }(y, s) \in B_{8 R} \backslash B_{2 M} \times(0, \infty) .
$$

By the same scaling argument as in the proof of Lemma 6.3, we get

$$
\left|\nabla_{y} \nu(y, s)\right| \lesssim \frac{R^{4} \lambda^{\gamma}}{|y|^{\frac{7}{2}}} \quad \text { for }(y, s) \in B_{6 R} \backslash B_{2 M} \times(0, \infty) .
$$

Next we consider the equation for $\partial_{y_{i}} \nu(y, s)$. We again use the same scaling argument as above to get

$$
\left|\partial_{y_{i}} \nu(y, s)\right|+|y| \cdot\left|\nabla_{y} \partial_{y_{i}} \nu(y, s)\right| \lesssim \frac{R^{4} \lambda^{\gamma}}{|y|^{\frac{7}{2}}} \quad \text { for }(y, s) \in B_{4 R} \backslash B_{2 M} \times(0, \infty) .
$$


We finally consider the equation for $\partial_{y_{j}} \partial_{y_{i}} \nu(y, s)$ and obtain

$$
\left|\partial_{y_{j}} \partial_{y_{i}} \nu(y, s)\right|+|y| \cdot\left|\nabla_{y} \partial_{y_{j}} \partial_{y_{i}} \nu(y, s)\right| \lesssim \frac{R^{4} \lambda^{\gamma}}{|y|^{\frac{9}{2}}} \quad \text { for }(y, s) \in B_{2 R} \backslash B_{2 M} \times(0, \infty) .
$$

Since the constant $M$ is independent of $R$, the proof is completed.

We now put

$$
\epsilon=-H_{y} E=-H_{y}\left(E_{1}+\nu+c(s) \psi_{1}^{(8 R)}\right) .
$$

Since $-H_{y} g=G_{\text {in }}$ for $|y|<2 R$, it is clear that $\epsilon(y, s)$ satisfies

$$
\begin{cases}\partial_{s} \epsilon=H_{y} \epsilon+G_{\text {in }} & \text { in } B_{2 R} \times(0, \infty) \\ \epsilon=\mathrm{d}_{\text {in }} \psi_{1}^{(8 R)} & \text { for } s=0 .\end{cases}
$$

From Lemma 6.2 - Lemma 6.4 and 6.13$)$, we conclude

$$
\begin{aligned}
|\epsilon(y, s)|+|y| \cdot\left|\nabla_{y} \epsilon(y, s)\right| & \lesssim \frac{\lambda^{\gamma}}{1+|y|}+\frac{R \lambda^{\gamma}}{1+|y|^{2}}+\frac{R^{4} \lambda^{\gamma}}{1+|y|^{\frac{9}{2}}}+\frac{R \lambda^{\gamma} e^{-\sqrt{\left|\mu_{1}\right|} \cdot|y|}}{1+|y|^{2}} \\
& \lesssim \frac{R^{4} \lambda^{\gamma}}{1+|y|^{\frac{9}{2}}} \quad \text { for }(y, s) \in B_{2 R} \times(0, \infty) .
\end{aligned}
$$

\section{Outer solution}

We now handle the outer solution $W(x, t)$. A goal of this section is to show $W(x, t) \in X_{\sigma}$. We recall that $W(x, t) \in X_{\sigma}$ is defined by

$$
W(x, t) \leq\left\{\begin{array}{ll}
\delta_{0}(T-t)^{l}\left(1+|z|^{2 l+2}\right) & \text { for }|z|<(T-t)^{-\frac{l}{2 l+2}}, \\
\frac{\delta_{0}}{1+|x|^{2}} & \text { for }|z|>(T-t)^{-\frac{l}{2 l+2}},
\end{array} \quad z=\frac{x}{\sqrt{T-t}} .\right.
$$

The case $l=0$ is treated in [6]. We here derive more elaborate decay estimates for the case $l \geq 1$ by using the method in [11, 12, 16, 18. Throughout this section, $\tilde{w}(x, t) \in C\left(\mathbb{R}^{5} \times[0, T]\right)$ represents an extension of $w(x, t) \in X_{\sigma}$ defined in Section 5.2 , and $(\lambda(t), \epsilon(y, t))$ represents a pair of functions obtained in Section 6 .

\subsection{Choice of parameters}

In this section, we consider

$$
\begin{cases}W_{t}=\Delta_{x} W+G_{\text {out }}(\lambda, \tilde{w}, \epsilon) & \text { in } \mathbb{R}^{5} \times(0, T), \\ W=(\mathbf{d} \cdot \mathbf{e}) \chi_{\mathrm{out}} & \text { for } t=0\end{cases}
$$

where $\mathbf{d}=\left(\mathrm{d}_{0}, \mathrm{~d}_{1}, \cdots, \mathrm{d}_{l}\right) \in \mathbb{R}^{l+1}$ is a parameter and

$$
\mathbf{e}=\left(e_{0}(z), e_{1}(z), \cdots, e_{l}(z)\right), \quad z=\frac{x}{\sqrt{T-t}} .
$$

We recall that $e_{i}(z)$ is the eigenfunction defined in Section 3.5. We introduce a self-similar transformation.

$$
\varphi(z, \tau)=W(z \sqrt{T-t}, t), \quad T-t=e^{-\tau} .
$$

The function $\varphi(z, \tau)$ solves

$$
\begin{cases}\varphi_{\tau}=A_{z} \varphi+e^{-\tau} G_{\text {out }}(\lambda, \tilde{w}, \epsilon) & \text { in } \mathbb{R}^{5} \times\left(\tau_{0}, \infty\right) \\ \varphi=(\mathbf{d} \cdot \mathbf{e}) \chi_{\text {out }} & \text { for } \tau=\tau_{0}=-\log T .\end{cases}
$$

We decompose the initial data to the subspace $Y_{l}=\operatorname{span}\left\{e_{0}, e_{1}, \cdots, e_{l}\right\}$ and its orthogonal complement in $L_{\rho}^{2}\left(\mathbb{R}^{5}\right)$.

$$
(\mathbf{d} \cdot \mathbf{e}) \chi_{\mathrm{out}}=\sum_{j, k=0}^{l} \frac{\mathrm{d}_{j}}{\kappa_{k}^{2}}\left(e_{j} \chi_{\mathrm{out}}, e_{k}\right) e_{k}+\left\{(\mathbf{d} \cdot \mathbf{e}) \chi_{\mathrm{out}}\right\}^{\perp}, \quad \kappa_{j}=\sqrt{\left(e_{j}, e_{j}\right)_{\rho}} .
$$

We define $\Phi(z, \tau)$ as

$$
\varphi=\mathbf{b}(\tau) \cdot \mathbf{e}+\Phi
$$


where $\mathbf{b}(\tau)=\left(\mathrm{b}_{0}(\tau), \mathrm{b}_{1}(\tau), \cdots, \mathrm{b}_{l}(\tau)\right) \in \mathbb{R}^{l+1}$. We easily see that $\Phi(z, \tau)$ satisfies

$$
\begin{cases}\Phi_{\tau}=A_{z} \Phi+e^{-\tau} G_{\text {out }}(\lambda, \tilde{w}, \epsilon)-\sum_{k=0}^{l} k \mathrm{~b}_{k} e_{k}-\sum_{k=0}^{l} \frac{d \mathrm{~b}_{k}}{d \tau} e_{k} \quad \text { in } \mathbb{R}^{5} \times\left(\tau_{0}, \infty\right), \\ \Phi=\left(\sum_{j, k=0}^{l} \frac{\mathrm{d}_{j}}{\kappa_{k}^{2}}\left(e_{j}, \chi_{\text {out }} e_{k}\right)-\sum_{k=0}^{l} \mathrm{~b}_{k}\left(\tau_{0}\right)\right) e_{k}+\left\{(\mathbf{d} \cdot \mathbf{e}) \chi_{\text {out }}\right\}^{\perp} & \text { for } \tau=\tau_{0} .\end{cases}
$$

To obtain a solution $\Phi(z, \tau)$ satisfying $\|\Phi(\tau)\|_{\rho}=o\left(e^{-l \tau}\right)$, we choose $\mathbf{b}(\tau)$ as

$$
\mathrm{b}_{k}(\tau)=-e^{-k \tau} \int_{\tau}^{\infty} \frac{e^{(k-1) \tau^{\prime}}}{\kappa_{k}^{2}}\left(G_{\text {out }}(\lambda, \tilde{w}, \epsilon), e_{k}\right)_{\rho} d \tau^{\prime}
$$

From Lemma 7.1, we verify that

$$
\begin{aligned}
\mid\left(G_{\text {out }}\right. & \left.(\lambda, \tilde{w}, \epsilon), e_{k}\right)_{\rho}|\lesssim|\left(G_{\text {out }}(\lambda, \tilde{w}, \epsilon), e_{k}\right)_{L_{\rho}^{2}(|z|<1)} \mid+e^{-2 l \tau} \\
& \lesssim \frac{e^{-l \tau}}{\lambda^{2} R^{\frac{1}{4}}}\left(\mathbf{1}_{|y|<2 R}+\frac{\mathbf{1}_{|y|>2 R}}{|y|^{\frac{11}{4}}},\left|e_{k}\right|\right)_{L_{\rho}^{2}(|z|<1)}+e^{-p l \tau}+e^{-2 l \tau} \\
& \lesssim R^{\frac{19}{4}} \lambda^{3} e^{-\left(l-\frac{5}{2}\right) \tau}+R^{-\frac{1}{4}} \lambda^{\frac{3}{4}} e^{-\left(l-\frac{11}{8}\right) \tau}+e^{-2 l \tau} \\
& \lesssim e^{-2 l \tau}
\end{aligned}
$$

This implies

$$
\left|\mathrm{b}_{k}(\tau)\right| \lesssim e^{-(2 l+1) \tau}
$$

From definition, the parameter $\mathbf{b}(\tau)$ gives a solution of

$$
\frac{d \mathrm{~b}_{k}}{d \tau}=-k \mathrm{~b}_{k}+\frac{e^{-\tau}}{\kappa_{k}^{2}}\left(G_{\text {out }}(\lambda, \tilde{w}(x, t), \epsilon(y, t)), e_{k}\right)_{\rho} .
$$

We take $\mathbf{d}=\left(\mathrm{d}_{0}, \mathrm{~d}_{1}, \cdots, \mathrm{d}_{l}\right)$ as

$$
(\mathrm{id}+\mathrm{C}) \mathbf{d}=\mathbf{b}\left(\tau_{0}\right),
$$

where $\mathrm{C}$ is a constant $(l+1) \times(l+1)$ matrix defined by

$$
\mathrm{C}_{k j}=\frac{1}{\kappa_{k}^{2}}\left(e_{j},\left.\left(1-\chi_{\mathrm{out}}\right)\right|_{\tau=\tau_{0}} e_{k}\right)_{\rho} .
$$

Since $\chi_{\text {out }}=\chi\left(\frac{|z|}{e^{B \tau}}\right)$ with $B=\frac{l+\frac{1}{2}}{2 l+2}$, we easily see that $\left|C_{k j}\right| \ll 1$, Therefore we get from 7.4 that

$$
|\mathbf{d}| \lesssim\left|\mathbf{b}\left(\tau_{0}\right)\right| \lesssim e^{-(2 l+1) \tau_{0}}
$$

By the choice of $\mathbf{d}$ and $\mathbf{b}(\tau)$, the equation 7.3 is rewritten as

$$
\begin{cases}\Phi_{\tau}=A_{z} \Phi+e^{-\tau} G_{\text {out }}^{\perp} & \text { in } \mathbb{R}^{5} \times\left(\tau_{0}, \infty\right), \\ \Phi=\left\{(\mathbf{d} \cdot \mathbf{e}) \chi_{\text {out }}\right\}^{\perp} & \text { for } \tau=\tau_{0}\end{cases}
$$

where $G_{\text {out }}^{\perp}=G_{\text {out }}(\lambda, \tilde{w}, \epsilon)-\sum_{k=0}^{l} \frac{e^{-\tau}}{\kappa_{k}^{2}}\left(G_{\text {out }}(\lambda, \tilde{w}, \epsilon), e_{k}\right)_{\rho} e_{k}$.

\subsection{Estimate of $G_{\text {out }}$}

We here provide the estimate of $G_{\text {out }}$. From (5.2), 5.4 and (5.7), we recall that

$$
G_{\text {out }}(\lambda, \tilde{w}, \epsilon)=h_{\text {out }}+h_{\text {in }}+\frac{1}{\lambda^{2}}\left(1-\chi_{\text {in }}\right) V(y)\left(\Theta \chi_{\text {out }}+\tilde{w}\right)+\frac{\lambda_{t}}{\lambda^{\frac{5}{2}}}\left(1-\chi_{\text {in }}\right) \Lambda_{y} \mathrm{Q}(y)+\mathrm{N}(v),
$$

where

$$
\begin{aligned}
& h_{\mathrm{out}}=2 \nabla_{x} \Theta \cdot \nabla_{x} \chi_{\mathrm{out}}+\Theta \Delta_{x} \chi_{\mathrm{out}}-\Theta \partial_{t} \chi_{\mathrm{out}}, \\
& h_{\mathrm{in}}=\frac{1}{\lambda^{\frac{7}{2}}}\left(2 \nabla_{y} \epsilon \cdot \nabla_{y} \chi_{\mathrm{in}}+\epsilon \Delta_{y} \chi_{\mathrm{in}}\right)+\frac{\lambda_{t}}{\lambda^{\frac{5}{2}}} \Lambda_{y} \epsilon \chi_{\mathrm{in}}-\frac{1}{\lambda^{\frac{3}{2}}} \epsilon \partial_{t} \chi_{\mathrm{in}}, \\
& \chi_{\mathrm{in}}=\chi\left(\frac{|y|}{R}\right), \\
& \chi_{\mathrm{out}}=\chi\left(\frac{|z|}{e^{B \tau}}\right) \quad \text { with } B=\frac{l+\frac{1}{2}}{2 l+2}, \\
& \mathrm{~N}(v)=f\left(\mathrm{Q}_{\lambda}+\Theta \chi_{\mathrm{out}}+\epsilon_{\lambda} \chi_{\mathrm{in}}+\tilde{w}\right)-f\left(\mathrm{Q}_{\lambda}\right)-f^{\prime}\left(\mathrm{Q}_{\lambda}\right)\left(\Theta \chi_{\mathrm{out}}+\epsilon_{\lambda} \chi_{\mathrm{in}}+\tilde{w}\right)
\end{aligned}
$$


and

$$
y=\frac{x}{\lambda}, \quad z=\frac{x}{\sqrt{T-t}}, \quad \lambda \sim \alpha_{l}^{2}(T-t)^{2 l+2} .
$$

Let $\mathbf{1}_{z \in \Omega}(z)$ be a function on $\mathbb{R}^{5}$ defined by $\mathbf{1}_{z \in \Omega}(z)=1$ if $z \in \Omega$ and $\mathbf{1}_{z \in \Omega}(z)=0$ if $z \notin \Omega$.

Lemma 7.1. Let $w \in X_{\sigma}$ and $(\tilde{w}(x, t), \lambda(t), \epsilon(y, t))$ be given in Section 6 . Then

$$
\mid G_{\text {out }}(\lambda, \tilde{w}, \epsilon) \lesssim \begin{cases}\frac{e^{-l \tau}}{\lambda^{2}} \frac{1}{R^{\frac{1}{4}}}\left(\frac{\mathbf{1}_{|y|<2 R}}{1+|y|^{\frac{9}{4}}}+\frac{1}{1+|y|^{\frac{11}{4}}}\right)+e^{-p l \tau} & \text { for }|z|<1, \\ e^{-2 l \tau}|z|^{4 l+4} & \text { for } 1<|z|<e^{\frac{l \tau}{2 l+2}}, \\ e^{-\left(l-\frac{1}{2 l+2}\right) \tau}|z|^{2 l+2} \mathbf{1} e^{\frac{\left(l+\frac{1}{2}\right) \tau}{2 l+2}}<|z|<2 e^{\frac{\left(l+\frac{1}{2}\right) \tau}{2 l+2}}+1 & \text { for } e^{\frac{l \tau}{2 l+2}}<|z|<e^{\frac{\tau}{2}}, \\ \frac{e^{-(3 l+2) \tau}}{|x|^{3}}+\frac{\delta_{0}^{2}}{|x|^{2 p}} & \text { for }|x|>1 .\end{cases}
$$

Proof. Since $\Theta(x, t)=-e^{-l \tau} e_{l}(z)$ and $B=e^{\frac{l+\frac{1}{2}}{2 l+2}}$, we see that

$$
\begin{aligned}
\left|h_{\text {out }}\right| & \lesssim\left|\frac{\partial z_{j}}{\partial x_{i}}\right| \cdot\left|\nabla_{z} \Theta \cdot \nabla_{z} \chi_{\text {out }}\right|+\left|\frac{\partial z_{j}}{\partial x_{i}}\right|^{2} \cdot\left|\Theta \Delta_{z} \chi_{\text {out }}\right|+\left|\frac{d \tau}{d t}\right| \cdot\left|\Theta \partial_{\tau} \chi_{\text {out }}\right| \\
& \lesssim e^{\tau} e^{-l \tau}|z|^{2 l} \mathbf{1}_{e^{B \tau}<|z|<2 e^{B \tau}} \\
& \lesssim e^{-\left(l-\frac{1}{2 l+2}\right) \tau}|z|^{2 l+2} \mathbf{1}_{e^{B \tau}<|z|<2 e^{B \tau}} .
\end{aligned}
$$

We next estimate $h_{\mathrm{in}}$. Since $\frac{e^{-(4 l+3) \tau}}{\lambda^{\frac{3}{2}}} \sim e^{-l \tau}$ and $\left|\lambda \lambda_{t}\right| \sim e^{-(4 l+3) \tau}$ (see Lemma 6.1), we get from (6.16) that

$$
\begin{aligned}
\left|h_{\mathrm{in}}\right| & \lesssim \frac{1}{\lambda^{\frac{7}{2}}}\left(\frac{\left|\nabla_{y} \epsilon\right|}{R}+\frac{|\epsilon|}{R^{2}}\right) \mathbf{1}_{R<|y|<2 R}+\frac{\left|\lambda_{t}\right|}{\lambda^{\frac{5}{2}}}\left|\Lambda_{y} \epsilon\right| \chi_{\mathrm{in}}+\frac{1}{\lambda^{\frac{3}{2}}}|\epsilon| \frac{\lambda_{t}}{\lambda} \mathbf{1}_{R<|y|<2 R} \\
& \lesssim \frac{1}{\lambda^{2}} \frac{1}{\lambda^{\frac{3}{2}}}\left(\frac{\left|\nabla_{y} \epsilon\right|}{R}+\frac{|\epsilon|}{R^{2}}+\left|\lambda \lambda_{t}\right| \cdot|\epsilon|\right) \mathbf{1}_{R<|y|<2 R}+\frac{1}{\lambda^{2}} \frac{\left|\lambda \lambda_{t}\right|}{\lambda^{\frac{3}{2}}}\left|\Lambda_{y} \epsilon\right| \chi_{\mathrm{in}} \\
& \lesssim \frac{e^{-l \tau}}{\lambda^{2}}\left(\frac{1}{R^{\frac{5}{2}}}+\frac{e^{-(4 l+3) \tau}}{R^{\frac{1}{2}}}\right) \mathbf{1}_{R<|y|<2 R}+\frac{e^{-l \tau}}{\lambda^{2}} \frac{R^{4} e^{-(4 l+3) \tau}}{1+|y|^{\frac{9}{2}}} \mathbf{1}_{|y|<2 R} .
\end{aligned}
$$

Therefore since $R=\tau_{0}$, we deduce that

$$
\begin{aligned}
\left|h_{\text {in }}\right| & \lesssim \frac{e^{-l \tau}}{\lambda^{2}} \frac{1}{R^{\frac{5}{2}}} \mathbf{1}_{R<|y|<2 R}+\frac{e^{-l \tau}}{\lambda^{2}} \frac{e^{-\tau}}{1+|y|^{\frac{9}{2}}} \mathbf{1}_{|y|<2 R} \\
& \lesssim \frac{e^{-l \tau}}{\lambda^{2}} \frac{1}{R^{\frac{1}{4}}} \frac{\mathbf{1}_{|y|<2 R}}{1+|y|^{\frac{9}{4}}} .
\end{aligned}
$$

We estimate the third term. Since $\tilde{w}(x, t)$ satisfies $(5.9)$, we verify that

$$
\begin{gathered}
\frac{1-\chi_{\text {in }}}{\lambda^{2}} V(y)\left|\Theta \chi_{\text {out }}+\tilde{w}\right| \lesssim \frac{e^{-l \tau} \mathbf{1}_{|y|>R}}{\lambda^{2}|y|^{4}}\left(\left(1+|z|^{2 l}\right) \chi_{\text {out }}+\delta_{0}\left(1+|z|^{2 l+2}\right) \mathbf{1}_{|z|<e^{\frac{l \tau}{2 l+2}}}\right) \\
\quad+\frac{1}{\lambda^{2}|y|^{4}} \frac{\mathbf{1}_{|z|>\frac{l \tau}{2+2}}}{1+|x|^{2}} \\
\lesssim \frac{e^{-l \tau}}{\lambda^{2}|y|^{4}}\left(\mathbf{1}_{|y|>R} \mathbf{1}_{|z|<1}+|z|^{2 l+2} \mathbf{1}_{1<|z|<2 e^{\frac{\left(l+\frac{1}{2}\right) \tau}{2 l+2}}}\right) \\
\quad+\frac{\lambda^{2} e^{2 \tau}}{|z|^{4}} \mathbf{1}_{e^{\frac{l \tau}{2 l+2}}<|z|<e^{\frac{\tau}{2}}}+\frac{\lambda^{2}}{|x|^{6}} \mathbf{1}_{|x|>1} \\
\lesssim \frac{e^{-l \tau}}{\lambda^{2}|y|^{4}} \mathbf{1}_{|y|>R} \mathbf{1}_{|z|<1}+e^{-(5 l+2) \tau}|z|^{2 l-2} \mathbf{1} \\
\quad+e^{-\left(4 l+4-\frac{2}{l+1}\right) \tau} \mathbf{1}_{e^{\frac{l \tau}{2 l+2}}<|z|<e^{\frac{\tau}{2}}}+\frac{e^{-(4 l+4) \tau}}{|x|^{6}} \mathbf{1}_{|x|>1} .
\end{gathered}
$$


The fourth term is easily estimated as

$$
\begin{aligned}
\left|\frac{\lambda_{t}}{\lambda^{\frac{5}{2}}}\left(1-\chi_{\text {in }}\right) \Lambda_{y} \mathrm{Q}(y)\right| & <\frac{1}{\lambda^{2}} \frac{\left|\lambda_{t}\right|}{\lambda^{\frac{3}{2}}} \frac{1}{|y|^{3}} \mathbf{1}_{|y|>R}<\frac{e^{-l \tau}}{\lambda^{2}} \frac{1}{|y|^{3}} \mathbf{1}_{|y|>R} \\
& <\frac{e^{-l \tau}}{\lambda^{2}} \frac{1}{|y|^{3}} \mathbf{1}_{|y|>R} \mathbf{1}_{|z|<1}+\lambda e^{\frac{3}{2} \tau} \frac{e^{-l \tau}}{|z|^{3}} \mathbf{1}_{|z|>1} \\
& <\frac{e^{-l \tau}}{\lambda^{2}} \frac{1}{|y|^{3}} \mathbf{1}_{|y|>R} \mathbf{1}_{|z|<1}+e^{-\left(3 l+\frac{1}{2}\right) \tau} \mathbf{1}_{1<|z|<e^{\frac{\tau}{2}}}+\frac{e^{-(3 l+2) \tau}}{|x|^{3}} \mathbf{1}_{|x|>1} .
\end{aligned}
$$

We finally estimate $\mathbf{N}(v)$. Since

$$
|| a+\left.b\right|^{p-1}(a+b)-|a|^{p-1} a-\left.p|a|^{p-1} b|\lesssim| a\right|^{p-2} b^{2}+|b|^{p} \quad \text { for } a, b \in \mathbb{R} \quad(p \geq 2)
$$

we get

$$
\begin{aligned}
|\mathrm{N}(v)| & =\left|f\left(\mathrm{Q}_{\lambda}+\Theta \chi_{\mathrm{out}}+\epsilon_{\lambda} \chi_{\mathrm{in}}+\tilde{w}\right)-f\left(\mathrm{Q}_{\lambda}\right)-f^{\prime}\left(\mathrm{Q}_{\lambda}\right)\left(\Theta \chi_{\mathrm{out}}+\epsilon_{\lambda} \chi_{\mathrm{in}}+\tilde{w}\right)\right| \\
\lesssim & f^{\prime \prime}\left(\mathrm{Q}_{\lambda}\right)\left(\Theta \chi_{\mathrm{out}}+\epsilon_{\lambda} \chi_{\mathrm{in}}+\tilde{w}\right)^{2}+f\left(\Theta \chi_{\mathrm{out}}+\epsilon_{\lambda} \chi_{\mathrm{in}}+\tilde{w}\right) \\
\lesssim & \frac{1}{\sqrt{\lambda}} \frac{\Theta^{2} \chi_{\mathrm{out}}+\epsilon_{\lambda}^{2} \chi_{\mathrm{in}}+\tilde{w}^{2}}{1+|y|}+|\Theta|^{p} \chi_{\mathrm{out}}+\left|\epsilon_{\lambda}\right|^{p} \chi_{\mathrm{in}}+|\tilde{w}|^{p} \\
\lesssim & \left(\frac{1}{\sqrt{\lambda}} \frac{\Theta^{2}+\tilde{w}^{2}}{1+|y|}+|\Theta|^{p}+|\tilde{w}|^{p}\right) \mathbf{1}_{|z|<1}+\left(\frac{1}{\sqrt{\lambda}} \frac{\epsilon_{\lambda}^{2}}{1+|y|}+\left|\epsilon_{\lambda}\right|^{p}\right) \mathbf{1}_{|z|<2 R} \\
& \quad+\left(\frac{1}{\sqrt{\lambda}} \frac{\Theta^{2}+\tilde{w}^{2}}{|y|}+|\Theta|^{p}+|\tilde{w}|^{p}\right) \mathbf{1}_{1<|z|<e^{\frac{l \tau}{2 l+2}}} \\
& \quad+\left(\frac{1}{\sqrt{\lambda}} \frac{\Theta^{2}}{|y|}+|\Theta|^{p}\right) \mathbf{1}_{e^{\frac{l \tau}{2 l+2}}<|z|<2 e^{\frac{\left(l+\frac{1}{2}\right) \tau}{2 l+2}}}+\left(\frac{1}{\sqrt{\lambda}} \frac{\tilde{w}^{2}}{|y|}+|\tilde{w}|^{p}\right) \mathbf{1}_{|z|>e^{\frac{l \tau}{2 l+2}}}
\end{aligned}
$$

Since $|\Theta(x, t)| \lesssim(T-t)^{l}\left(1+|z|^{2 l}\right)$ and $\tilde{w}(x, t)$ satisfies $[5.9$, we see that

$$
\begin{aligned}
\left(\frac{1}{\sqrt{\lambda}}\right. & \left.\frac{\Theta^{2}+\tilde{w}^{2}}{1+|y|}+|\Theta|^{p}+|\tilde{w}|^{p}\right) \mathbf{1}_{|z|<1} \lesssim\left(\frac{1}{\sqrt{\lambda}} \frac{e^{-2 l \tau}}{1+|y|}+e^{-p l \tau}\right) \mathbf{1}_{|z|<1} \\
& \lesssim \frac{e^{-2 l \tau}}{\sqrt{\lambda}}\left(\mathbf{1}_{|y|<1}+\frac{|y|^{\frac{7}{4}}}{|y|^{\frac{11}{4}}} \mathbf{1}_{|y|>1} \mathbf{1}_{|z|<1}\right)+e^{-p l \tau} \mathbf{1}_{|z|<1} .
\end{aligned}
$$

Here we note that $|y|^{\frac{7}{4}} \mathbf{1}_{|z|<1}=\left(\lambda^{-1} e^{-\frac{\tau}{2}}\right)^{\frac{7}{4}}|z|^{\frac{7}{4}} \mathbf{1}_{|z|<1}<\left(\lambda^{-1} e^{-\frac{\tau}{2}}\right)^{\frac{7}{4}} \mathbf{1}_{|z|<1}$. Therefore we deduce that

$$
\begin{aligned}
\left(\frac{1}{\sqrt{\lambda}} \frac{\Theta^{2}+\tilde{w}^{2}}{1+|y|}+|\Theta|^{p}+|\tilde{w}|^{p}\right) \mathbf{1}_{|z|<1} & \lesssim \frac{e^{-2 l \tau}}{\sqrt{\lambda}}\left(1+\frac{e^{-\frac{7 \tau}{8}}}{\lambda^{\frac{7}{4}}} \frac{1}{|y|^{\frac{11}{4}}} \mathbf{1}_{|y|>1}\right) \mathbf{1}_{|z|<1}+e^{-p l \tau} \mathbf{1}_{|z|<1} \\
& \lesssim \frac{1}{\lambda^{2}} \frac{e^{-\left(\frac{3 l}{2}+\frac{3}{8}\right) \tau}}{1+|y|^{\frac{11}{4}}} \mathbf{1}_{|z|<1}+e^{-p l \tau} \mathbf{1}_{|z|<1} .
\end{aligned}
$$

Furthermore from 6.16 and $\tau_{0}=R$, we verify that

$$
\begin{aligned}
\left(\frac{1}{\sqrt{\lambda}} \frac{\epsilon_{\lambda}^{2}}{1+|y|}+\left|\epsilon_{\lambda}\right|^{p}\right) \mathbf{1}_{|y|<2 R} & \lesssim \frac{1}{\lambda^{2}}\left(\frac{\lambda^{\frac{3}{2}}}{1+|y|} \frac{R^{8} e^{-2 l \tau}}{1+|y|^{9}}+\frac{\lambda^{2} R^{4 p} e^{-p l \tau}}{1+|y|^{\frac{9 p}{2}}}\right) \mathbf{1}_{|y|<2 R} \\
& \lesssim \frac{e^{-l \tau}}{\lambda^{2}}\left(\frac{R^{8} e^{-(4 l+3) \tau}}{1+|y|^{10}}+\frac{R^{4 p} e^{-((p+3) l+4) \tau}}{1+|y|^{\frac{9 p}{2}}}\right) \mathbf{1}_{|y|<2 R} \\
& \lesssim \frac{e^{-l \tau}}{\lambda^{2}}\left(\frac{e^{-\tau}}{1+|y|^{10}}+\frac{e^{-\tau}}{1+|y|^{\frac{9 p}{2}}}\right) \mathbf{1}_{|y|<2 R} .
\end{aligned}
$$

For the case $2<p<3$, there exists $c_{p}>0$ such that

$$
|\xi|^{p}<c_{p}\left(|\xi|^{2}+|\xi|^{3}\right) \quad \text { for } \xi \in \mathbb{R} .
$$


From this relation, we see that

$$
\begin{aligned}
\left(\frac{1}{\sqrt{\lambda}}\right. & \left.\frac{\Theta^{2}+\tilde{w}^{2}}{|y|}+|\Theta|^{p}+|\tilde{w}|^{p}\right) \mathbf{1}_{1<|z|<e^{\frac{l \tau}{2 l+2}}} \\
& \lesssim\left(\frac{1}{\sqrt{\lambda}} \frac{e^{-2 l \tau}\left(|z|^{4 l}+\delta_{0}^{2}|z|^{4 l+4}\right)}{|y|}+e^{-p l \tau}\left(|z|^{2 p l}+\delta_{0}^{p}|z|^{2 p(l+1)}\right)\right) \mathbf{1}_{1<|z|<e^{\frac{l \tau}{2 l+2}}} \\
& \lesssim\left(\frac{1}{\sqrt{\lambda}} \frac{e^{-2 l \tau}|z|^{4 l+4}}{|y|}+\left(e^{-l \tau}|z|^{2(l+1)}\right)^{p}\right) \mathbf{1}_{1<|z|<e^{\frac{l \tau}{2 l+2}}} \\
& \lesssim\left(\frac{\sqrt{\lambda} e^{\frac{\tau}{2}}}{|z|} e^{-2 l \tau}|z|^{4 l+4}+e^{-2 l \tau}|z|^{4(l+1)}+e^{-3 l \tau}|z|^{6(l+1)}\right) \mathbf{1}_{1<|z|<e^{\frac{l \tau}{2 l+2}}} \\
& \lesssim e^{-2 l \tau}|z|^{4 l+4} \mathbf{1}_{1<|z|<e^{\frac{l \tau}{2 l+2}}} .
\end{aligned}
$$

We use 7.11 again to get

$$
\begin{aligned}
& \left(\frac{1}{\sqrt{\lambda}} \frac{\Theta^{2}}{|y|}+|\Theta|^{p}\right) \mathbf{1}_{e^{\frac{l \tau}{2 l+2}}<|z|<2 e^{\frac{\left(l+\frac{1}{2}\right) \tau}{2 l+2}}} \lesssim\left(\frac{1}{\sqrt{\lambda}} \frac{e^{-2 l \tau}|z|^{4 l}}{|y|}+e^{-p l \tau}|z|^{2 p l}\right) \mathbf{1}_{e^{\frac{l \tau}{2 l+2}}<|z|<2 e^{\frac{\left(l+\frac{1}{2}\right) \tau}{2 l+2}}} \\
& \lesssim\left(e^{-2 l \tau}|z|^{4 l}+e^{-3 l \tau}|z|^{6 l}\right) \mathbf{1}_{e^{\frac{l \tau}{2 l+2}}<|z|<2 e^{\frac{\left(l+\frac{1}{2}\right) \tau}{2 l+2}}} \\
& \lesssim e^{-2 l \tau}|z|^{4 l} \mathbf{1} e^{\frac{l \tau}{2 l+2}}<|z|<2 e^{\frac{\left(l+\frac{1}{2}\right) \tau}{2 l+2}} \\
& \lesssim e^{-(l+1) \tau}|z|^{2 l+2} \mathbf{1} e^{\frac{l \tau}{2 l+2}}<|z|<2 e^{\frac{\left(l+\frac{1}{2}\right) \tau}{2 l+2}} .
\end{aligned}
$$

We finally estimate the last term.

$$
\begin{aligned}
\left(\frac{1}{\sqrt{\lambda}} \frac{\tilde{w}^{2}}{|y|}+|\tilde{w}|^{p}\right) \mathbf{1}_{|z|>e^{\frac{l \tau}{2 l+2}}} & \lesssim\left(\frac{1}{\sqrt{\lambda}} \frac{1}{|y|} \frac{\delta_{0}^{2}}{1+|x|^{4}}+\frac{\delta_{0}^{p}}{1+|x|^{2 p}}\right) \mathbf{1}_{|z|>e^{\frac{l \tau}{2 l+2}}} \\
& \lesssim\left(\frac{\sqrt{\lambda} e^{\frac{\tau}{2}}}{|z|}+1\right) \mathbf{1}_{e^{\frac{l \tau}{2 l+2}}<|z|<e^{\frac{\tau}{2}}}+\left(\frac{\sqrt{\lambda}}{|x|} \frac{\delta_{0}^{2}}{|x|^{4}}+\frac{\delta_{0}^{p}}{|x|^{2 p}}\right) \mathbf{1}_{|x|>1} \\
& \lesssim \mathbf{1}_{e^{\frac{l \tau}{2 l+2}}<|z|<e^{\frac{\tau}{2}}}+\frac{\delta_{0}^{2}}{|x|^{2 p}} \mathbf{1}_{|x|>1} .
\end{aligned}
$$

From the above estimates, we conclude that

$$
\begin{aligned}
|\mathrm{N}(v)| \lesssim & \frac{e^{-l \tau}}{\lambda^{2}} \frac{e^{-\frac{3}{8} \tau}}{1+|y|^{\frac{11}{4}}} \mathbf{1}_{|z|<1}+e^{-p l \tau} \mathbf{1}_{|z|<1}+e^{-2 l \tau}|z|^{4 l+4} \mathbf{1}_{1<|z|<e^{\frac{l \tau}{2 l+2}}} \\
& +e^{-(l+1) \tau}|z|^{2 l+2} \mathbf{1}_{e^{\frac{l \tau}{2 l+2}}<|z|<2 e^{\frac{\left(l+\frac{1}{2}\right) \tau}{2 l+2}}}+\mathbf{1}_{e^{\frac{l \tau}{2 l+2}}<|z|<e^{\frac{\tau}{2}}}+\frac{\delta_{0}^{2}}{|x|^{2 p}} \mathbf{1}_{|x|>1} .
\end{aligned}
$$

Combining 7.7 - 7.10 and 7.12, we complete the proof.

\section{3 $L_{\rho}^{2}\left(\mathbb{R}^{5}\right)$ estimate for $\Phi(z, \tau)$}

To derive the estimate for a solution $\Phi(z, \tau)$ of 7.6 , we first consider

$$
\begin{cases}\partial_{\tau} \Phi_{1}=A_{z} \Phi_{1} & \text { in } \mathbb{R}^{5} \times\left(\tau_{0}, \infty\right), \\ \Phi_{1}=\left\{(\mathbf{d} \cdot \mathbf{e}) \chi_{\mathrm{out}}\right\}^{\perp} & \text { for } \tau=\tau_{0} .\end{cases}
$$

Lemma 7.2. There exists $K_{1}>1$ independent of $R, \delta_{0}, \sigma$ such that

$$
\left|\Phi_{1}(z, \tau)\right|<K_{1} e^{-(l+1) \tau}\left(1+|z|^{2 l+2}\right) \quad \text { for }(z, \tau) \in \mathbb{R}^{5} \times\left(\tau_{0}, \infty\right) .
$$


Proof. We estimate the initial data.

$$
\begin{aligned}
\left\{(\mathbf{d} \cdot \mathbf{e}) \chi_{\mathrm{out}}\right\}^{\perp} & =(\mathbf{d} \cdot \mathbf{e}) \chi_{\mathrm{out}}-\sum_{j, k=0}^{l} \frac{\mathrm{d}_{j}}{\kappa_{k}^{2}}\left(e_{j} \chi_{\mathrm{out}}, e_{k}\right)_{\rho} e_{k} \\
& =(\mathbf{d} \cdot \mathbf{e}) \chi_{\mathrm{out}}-\sum_{j, k=0}^{l} \frac{\mathrm{d}_{j}}{\kappa_{k}^{2}}\left(e_{j}, e_{k}\right)_{\rho} e_{k}+\sum_{j, k=0}^{l} \frac{\mathrm{d}_{j}}{\kappa_{k}^{2}}\left(e_{j}\left(1-\chi_{\mathrm{out}}\right), e_{k}\right)_{\rho} e_{k} \\
& =(\mathbf{d} \cdot \mathbf{e})\left(\chi_{\mathrm{out}}-1\right)+\sum_{j, k=0}^{l} \frac{\mathrm{d}_{j}}{\kappa_{k}^{2}}\left(e_{j}\left(1-\chi_{\mathrm{out}}\right), e_{k}\right)_{\rho} e_{k} .
\end{aligned}
$$

Since $\chi_{\mathrm{out}}=\chi\left(\frac{|z|}{e^{B \tau}}\right)$ with $B=\frac{l+\frac{1}{2}}{2 l+2}$, we see from 7.5 that

$$
\left\|\left\{(\mathbf{d} \cdot \mathbf{e}) \chi_{\text {out }}\right\}^{\perp}\right\|_{\rho} \lesssim|\mathbf{d}| \lesssim e^{-(2 l+1) \tau_{0}} .
$$

Therefore we deduce that

$$
\left\|\Phi_{1}(\tau)\right\|_{\rho} \lesssim\left\|\left\{(\mathbf{d} \cdot \mathbf{e}) \chi_{\text {out }}\right\}^{\perp}\right\|_{\rho} e^{-(l+1)\left(\tau-\tau_{0}\right)} \lesssim e^{-(l+1) \tau} .
$$

We next derive a pointwise estimate. Let $e_{l+1}(z)$ be given in Section 3.5 , which is written as

$$
e_{l+1}(z)=1+a_{1}|z|^{2}+a_{2}|z|^{4}+\cdots+a_{l+1}|z|^{2 l+2} .
$$

To construct a comparison function, we define

$$
\tilde{e}_{l+1}(z)=k e_{l+1}(z) \quad \text { with } \quad k= \begin{cases}1 & \text { if } a_{l+1}>0 \\ -1 & \text { if } a_{l+1}<0\end{cases}
$$

From this definition, there exists $r_{l+1}>0$ such that

$$
\tilde{e}_{l+1}(z)>\frac{\left|a_{l+1}\right|}{2}|z|^{2 l+2} \quad \text { for }|z|>r_{l+1} .
$$

Therefore we note from $(7.13)$ that there exists $K>1$ such that $\left|\Phi_{1}(z, \tau)\right|<K e^{-(l+1) \tau} \tilde{e}_{l+1}(z)$ for $|z|=r_{l+1}$. Furthermore it holds from 7 7.5 that $\left|\Phi_{1}\left(\tau_{0}\right)\right|<|\mathbf{d}| \cdot|\mathbf{e}(z)| \lesssim e^{-(2 l+1) \tau_{0}}|z|^{2 l}$ for $|z|>r_{l+1}$. Therefore a comparison argument shows that there exists $K^{\prime}>1$ such that

$$
\left|\Phi_{1}(z, \tau)\right|<K^{\prime} e^{-(l+1) \tau} \tilde{e}_{l+1}(z) \quad \text { for }|z|>r_{l+1}, \tau>\tau_{0} .
$$

This completes the proof.

Next we write $\Phi$ as

The function $\Phi_{2}(z, \tau)$ solves

$$
\Phi=\Phi_{1}+\Phi_{2}
$$

$$
\begin{cases}\partial_{\tau} \Phi_{2}=A_{z} \Phi_{2}+e^{-\tau} G_{\text {out }}^{\perp} & \text { in } \mathbb{R}^{5} \times\left(\tau_{0}, \infty\right), \\ \Phi_{2}=0 & \text { for } \tau=\tau_{0} .\end{cases}
$$

We first provide $L_{\rho}^{2}$ estimates of $G_{\text {out }}$.

Lemma 7.3. It holds that

$$
\left\|G_{\text {out }}\right\|_{\rho} \lesssim e^{-\left(2 l-\frac{1}{2}\right) \tau}
$$

Proof. From Lemma 7.1, we easily verify that

$$
\begin{aligned}
\left\|G_{\text {out }}\right\|_{\rho} & \lesssim\left\|G_{\text {out }} \mathbf{1}_{|z|<1}\right\|_{\rho}+\left\|G_{\text {out }} \mathbf{1}_{|z|>1}\right\|_{\rho} \\
& \lesssim\left\|G_{\text {out }} \mathbf{1}_{|z|<1}\right\|_{\rho}+e^{-2 l \tau}
\end{aligned}
$$

We estimate the first term.

$$
\begin{aligned}
\left\|G_{\text {out }} \mathbf{1}_{|z|<1}\right\|_{\rho} \lesssim & \frac{e^{-l \tau}}{\lambda^{2} R^{\frac{1}{4}}}\left(\left\|\frac{\mathbf{1}_{|y|<2 R}}{1+|y|^{\frac{9}{4}}}\right\|_{L^{2}(|z|<1)}+\left\|\frac{\mathbf{1}_{|y|<2 R}+\mathbf{1}_{|y|>2 R}}{1+|y|^{\frac{11}{4}}}\right\|_{L^{2}(|z|<1)}\right)+e^{-p l \tau} \\
& \lesssim \frac{e^{-l \tau}}{\lambda^{2} R^{\frac{1}{4}}}\left(\left(R \lambda e^{\frac{\tau}{2}}\right)^{\frac{5}{2}}+\left\|\frac{\mathbf{1}_{|y|>2 R}}{|y|^{\frac{11}{4}}}\right\|_{L^{2}(|z|<1)}\right)+e^{-p l \tau} \\
& \lesssim \frac{e^{-l \tau}}{\lambda^{2} R^{\frac{1}{4}}}\left(\left(R \lambda e^{\frac{\tau}{2}}\right)^{\frac{5}{2}}+\left(\lambda e^{\frac{\tau}{2}}\right)^{\frac{11}{4}}\left(R \lambda e^{\frac{\tau}{2}}\right)^{-\frac{1}{4}}\right)+e^{-p l \tau} \\
& \lesssim R^{\frac{9}{4}} e^{-\frac{\tau_{0}}{4}} e^{-\left(2 l-\frac{1}{2}\right) \tau}+e^{-p l \tau}
\end{aligned}
$$


Since $\tau_{0}=R$, we complete the proof.

From this estimates, we immediately obtain $L_{\rho}^{2}$ estimates of $\Phi_{2}(z, \tau)$.

Lemma 7.4. There exists $K_{2}>1$ independent of $R, \delta_{0}, \sigma$ such that

$$
\left\|\Phi_{2}(\tau)\right\|_{\rho}<K_{2} e^{-(l+1) \tau} .
$$

Proof. We take the inner product $\left(\cdot, \Phi_{2}\right)_{\rho}$ in 7.14 to get

$$
\frac{1}{2} \frac{d}{d \tau}\left\|\Phi_{2}\right\|_{\rho}^{2}=\left(A_{z} \Phi_{2}, \Phi_{2}\right)_{\rho}+e^{-\tau}\left(G_{\text {out }}^{\perp}, \Phi_{2}\right)_{\rho} .
$$

Since $\left(A_{z} \Phi_{2}, \Phi_{2}\right)<-(l+1)\left\|\Phi_{2}\right\|_{\rho}^{2}$, we deduce from Lemma 7.3 that

$$
\left\|\Phi_{2}(\tau)\right\|_{\rho} \lesssim e^{-(l+1) \tau}
$$

The proof is completed.

\subsection{Pointwise estimate for $\Phi_{2}(z, \tau)$ in $|z|<e^{\frac{l \tau}{2 l+2}}$}

Since $\Phi_{2}(z, \tau)$ is a solution of 7.14 , it is written as an integral form.

$$
\Phi_{2}(z, \tau)=\int_{\tau_{0}}^{\tau} e^{A_{z}\left(\tau-\tau^{\prime}\right) e^{-\tau^{\prime}}} G_{\text {out }}^{\perp} d \tau^{\prime}
$$

We here estimate $\Phi_{2}(z, \tau)$ by the same manner as in [11, 12] (see also [16, 18, and Section 5.2 [10]). For simplicity we put

$$
b=\min \left\{\frac{2 l+\frac{3}{2}}{4}, \frac{2 l+1}{2 l+2}\right\} .
$$

Proposition 7.1. There exists $K_{3}>1$ independent of $R, \delta_{0}, \sigma$ such that

$$
\begin{aligned}
& \int_{\tau_{0}}^{\tau} e^{A_{z}\left(\tau-\tau^{\prime}\right)} e^{-\tau^{\prime}}\left|G_{\text {out }}^{\perp}\right| d \tau^{\prime}< K_{3}\left(\frac{1}{R^{\frac{1}{4}}} \frac{e^{-l \tau}}{1+|y|^{\frac{1}{4}}}+T^{b} e^{-l \tau}+\frac{e^{-l \tau}}{R^{\frac{1}{4}}}|z|^{-2 l}+e^{-(2 l+1) \tau}|z|^{4 l+4}\right. \\
&\left.+e^{-\left(l+\frac{2 l+1}{2 l+2}\right) \tau}|z|^{2 l+2}\right) \quad \text { for }(z, \tau) \in \mathbb{R}^{n} \times\left(\tau_{0}, \infty\right) .
\end{aligned}
$$

As a consequence of this proposition, we obtain

$$
\begin{aligned}
\int_{\tau_{0}}^{\tau} e^{A_{z}\left(\tau-\tau^{\prime}\right)} e^{-\tau^{\prime}}\left|G_{\text {out }}^{\perp}\right| d \tau^{\prime} \lesssim & \frac{1}{R^{\frac{1}{4}}} \frac{e^{-l \tau}}{1+|y|^{\frac{1}{4}}}+T^{b} e^{-l \tau}+\frac{e^{-l \tau}}{R^{\frac{1}{4}}}|z|^{-2 l} \\
& +e^{-\left(l+\frac{2 l+1}{2 l+2}\right) \tau}|z|^{2 l+2} \quad \text { for }|z|^{\frac{l \tau}{2 l+2}}, \tau \in\left(\tau_{0}, \infty\right) .
\end{aligned}
$$

For simplicity of computation, we arrange the estimate in Lemma 7.1 as

$$
\begin{aligned}
\left|G_{\text {out }}\right| & \lesssim \frac{e^{-l \tau}}{\lambda^{2}} \frac{1}{R^{\frac{1}{4}}} \frac{1}{1+|y|^{\frac{9}{4}}}+\left(e^{-p l \tau}+e^{-2 l \tau}|z|^{4 l+4}+e^{-\left(l-\frac{1}{2 l+2}\right) \tau}|z|^{2 l+2}\right) \quad \text { for } z \in \mathbb{R}^{5} \\
& =: G_{\text {out }}^{(1)}+G_{\text {out }}^{(2)}
\end{aligned}
$$

We first prepare the following lemma.

Lemma 7.5. There exists $k>0$ independent of $\tau_{1}, R, \delta_{0}, \sigma$ such that

$$
\begin{aligned}
\int_{\tau_{1}}^{\tau} e^{A_{z}\left(\tau-\tau^{\prime}\right)} e^{-\tau^{\prime}}\left|G_{\text {out }}^{\perp}\right| d \tau^{\prime}<k & \left(\frac{1}{R^{\frac{1}{4}}} \frac{e^{-l \tau_{1}}}{1+|y|^{\frac{1}{4}}}+T^{b} e^{-l \tau_{1}}+e^{-(2 l+1) \tau}|z|^{4 l+4}\right. \\
& \left.+e^{-\left(l+\frac{2 l+1}{2 l+2}\right) \tau}|z|^{2 l+2}\right) \quad \text { for }(z, \tau) \in \mathbb{R}^{5} \times\left(\tau_{1}, \infty\right) .
\end{aligned}
$$


Proof. We write the integral as

$$
\begin{aligned}
\int_{\tau_{1}}^{\tau} e^{A_{z}\left(\tau-\tau^{\prime}\right)} e^{-\tau^{\prime}}\left|G_{\text {out }}^{\perp}\right| d \tau^{\prime} \lesssim & \int_{\tau_{1}}^{\tau} e^{A_{z}\left(\tau-\tau^{\prime}\right)} e^{-\tau^{\prime}}\left(\left|G_{\text {out }}^{(1)}\right|+\left|G_{\text {out }}^{(2)}\right|\right) d \tau^{\prime} \\
& +\sum_{k=0}^{l} \int_{\tau_{1}}^{\tau}\left|e^{A_{z}\left(\tau-\tau^{\prime}\right)} \frac{e^{-\tau^{\prime}}}{\kappa_{k}^{2}}\left(G_{\text {out }}, e_{k}\right)_{\rho} e_{k}\right| d \tau^{\prime} .
\end{aligned}
$$

From Lemma 3.7, we see that

$$
\begin{aligned}
\int_{\tau_{1}}^{\tau} e^{A_{z}\left(\tau-\tau^{\prime}\right)} e^{-\tau^{\prime}}\left|G_{\text {out }}^{(2)}\right| d \tau^{\prime} & \lesssim \int_{\tau_{1}}^{\tau}\left\{e^{-(p l+1) \tau^{\prime}}+e^{-(2 l+1) \tau^{\prime}}\left(1+e^{-(2 l+2)\left(\tau-\tau^{\prime}\right)}|z|^{4 l+4}\right)\right. \\
& \left.+e^{-\left(l+\frac{2 l+1}{2 l+2}\right) \tau^{\prime}}\left(1+e^{-(l+1)\left(\tau-\tau^{\prime}\right)}|z|^{2 l+2}\right)\right\} d \tau^{\prime} \\
& \lesssim e^{-\left(l+\frac{2 l+1}{2 l+2}\right) \tau_{1}}+e^{-(2 l+1) \tau}|z|^{4 l+4}+e^{-\left(l+\frac{2 l+1}{2 l+2}\right) \tau}|z|^{2 l+2} \\
& \lesssim T^{\frac{2 l+1}{2 l+2}} e^{-l \tau_{1}}+e^{-(2 l+1) \tau}|z|^{4 l+4}+e^{-\left(l+\frac{2 l+1}{2 l+2}\right) \tau}|z|^{2 l+2}
\end{aligned}
$$

Since $|a|^{k} \lesssim 1+|a|^{l+1}$ for $k=0,1,2, \cdots, l$, we get from Lemma 7.3 that

$$
\begin{aligned}
\int_{\tau_{1}}^{\tau}\left|e^{A_{z}\left(\tau-\tau^{\prime}\right)} e^{-\tau^{\prime}}\left(G_{\text {out }}, e_{k}\right)_{\rho} e_{k}\right| d \tau^{\prime} & =\int_{\tau_{1}}^{\tau}\left|e^{-k\left(\tau-\tau^{\prime}\right)} e^{-\tau^{\prime}}\left(G_{\text {out }}, e_{k}\right)_{\rho} e_{k}\right| d \tau^{\prime} \\
& \lesssim e^{-\left(2 l+\frac{1}{2}\right) \tau_{1}}\left(e^{-\left(\tau-\tau_{1}\right)}\left(1+|z|^{2}\right)\right)^{k} \\
& \lesssim e^{-\left(2 l+\frac{1}{2}\right) \tau_{1}}\left\{1+\left(e^{-\left(\tau-\tau_{1}\right)}\left(1+|z|^{2}\right)\right)^{l+1}\right\} \\
& \lesssim e^{-\left(2 l+\frac{1}{2}\right) \tau_{1}}+e^{-\left(l-\frac{1}{2}\right) \tau_{1}} e^{-(l+1) \tau}|z|^{2 l+2}
\end{aligned}
$$

Furthermore Lemma 3.8 implies

$$
\int_{\tau_{1}}^{\tau} e^{A_{z}\left(\tau-\tau^{\prime}\right)} e^{-\tau^{\prime}}\left|G_{\text {out }}^{(1)}\right| d \tau^{\prime} \lesssim \frac{1}{R^{\frac{1}{4}}} \frac{e^{-l \tau_{1}}}{1+|y|^{\frac{1}{4}}}+T^{\left(2 l+\frac{3}{2}\right) \frac{1}{4}} e^{-l \tau_{1}} .
$$

The proof is completed.

To obtain the estimate in Proposition 7.1, we consider four cases separately.

$$
\begin{aligned}
& \text { (i) } z \in \mathbb{R}^{n} \text { and } \tau \in\left(\tau_{0}, \tau_{0}+1\right), \\
& \text { (ii) }|z|<4 \text { and } \tau \in\left(\tau_{0}+1, \infty\right), \\
& \text { (iii) } 2<|z|<e^{\frac{\tau-\tau_{0}}{2}} \text { and } \tau \in\left(\tau_{0}+1, \infty\right), \\
& \text { (iv) }|z|>e^{\frac{\tau-\tau_{0}}{2}} \text { and } \tau \in\left(\tau_{0}+1, \infty\right) .
\end{aligned}
$$

\subsection{1 (i) Estimate in $z \in \mathbb{R}^{n}$ and $\tau \in\left(\tau_{0}, \tau_{0}+1\right)$}

In this case, the estimate follows from Lemma 7.5 .

7.4.2 (ii) Estimate in $|z|<4$ and $\tau \in\left(\tau_{0}+1, \infty\right)$

We divide the integral in 7.15 into two parts.

$$
\Phi_{2}(\tau)=\int_{\tau_{0}}^{\tau-1} e^{A_{z}\left(\tau-\tau^{\prime}\right)} e^{-\tau^{\prime}} G_{\text {out }}^{\perp} d \tau^{\prime}+\int_{\tau-1}^{\tau} e^{A_{z}\left(\tau-\tau^{\prime}\right)} e^{-\tau^{\prime}} G_{\text {out }}^{\perp} d \tau^{\prime} .
$$


We apply Lemma 3.6 and Lemma 7.3 to get

$$
\begin{aligned}
\left|\int_{\tau_{0}}^{\tau-1} e^{A_{z}\left(\tau-\tau^{\prime}\right)} e^{-\tau^{\prime}} G_{\text {out }}^{\perp} d \tau^{\prime}\right| & \lesssim \int_{\tau_{0}}^{\tau-1}\left|e^{A_{z} \frac{1}{2}} e^{A_{z}\left(\tau-\tau^{\prime}-\frac{1}{2}\right)} e^{-\tau^{\prime}} G_{\text {out }}^{\perp}\right| d \tau^{\prime} \\
& \lesssim \int_{\tau_{0}}^{\tau-1} \frac{e^{-\frac{1}{2}}|z|^{2}}{\frac{4\left(1+e^{-\frac{1}{2}}\right)}{\left(1-e^{-\frac{1}{2}}\right)^{\frac{n}{4}}}}\left\|e^{A_{z}\left(\tau-\tau^{\prime}-\frac{1}{2}\right)} e^{-\tau^{\prime}} G_{\text {out }}^{\perp}\right\|_{\rho} d \tau^{\prime} \\
& \lesssim \int_{\tau_{0}}^{\tau-1} e^{-(l+1)\left(\tau-\tau^{\prime}\right)}\left\|e^{-\tau^{\prime}} G_{\text {out }}^{\perp}\right\|_{\rho} d \tau^{\prime} \\
& \lesssim \int_{\tau_{0}}^{\tau-1} e^{-(l+1)\left(\tau-\tau^{\prime}\right)} e^{-\left(2 l+\frac{1}{2}\right) \tau^{\prime}} d \tau^{\prime} \\
& \lesssim e^{-(l+1) \tau} \quad \text { for }|z|<4, \tau \in\left(\tau_{0}+1, \infty\right)
\end{aligned}
$$

The second integral is reduced to Lemma 7.5

\subsection{3 (iii) Estimate in $2<|z|<e^{\frac{\tau-\tau_{0}}{2}}$ and $\tau \in\left(\tau_{0}+1, \infty\right)$}

For $2<|z|<e^{\frac{\tau-\tau_{0}}{2}}$, we define $\tau_{1} \in\left(\tau_{0}, \tau-1\right)$ by

$$
|z|=e^{\frac{\tau-\tau_{1}}{2}}
$$

We write $\Phi_{2}(z, \tau)$ as

$$
\Phi_{2}(\tau)=\int_{\tau_{0}}^{\tau_{1}} e^{A_{z}\left(\tau-\tau^{\prime}\right)} e^{-\tau^{\prime}} G_{\text {out }}^{\perp} d \tau^{\prime}+\int_{\tau_{1}}^{\tau-1} e^{A_{z}\left(\tau-\tau^{\prime}\right)} e^{-\tau^{\prime}} G_{\text {out }}^{\perp} d \tau^{\prime}+\int_{\tau-1}^{\tau} e^{A_{z}\left(\tau-\tau^{\prime}\right)} e^{-\tau^{\prime}} G_{\text {out }}^{\perp} d \tau^{\prime} .
$$

Since $\tau_{0}<\tau_{1}<\tau-1$ and $|z|=e^{\frac{\tau-\tau_{1}}{2}}$, we get from Lemma 3.6 and Lemma 7.3 that

$$
\begin{aligned}
\left|\int_{\tau_{0}}^{\tau_{1}} e^{A_{z}\left(\tau-\tau^{\prime}\right)} e^{-\tau^{\prime}} G_{\text {out }}^{\perp} d \tau^{\prime}\right| & \lesssim \int_{\tau_{0}}^{\tau_{1}}\left|e^{A_{z}\left(\tau-\tau_{1}\right)} e^{A_{z}\left(\tau-\tau^{\prime}+\tau_{1}\right)} e^{-\tau^{\prime}} G_{\text {out }}^{\perp}\right| d \tau^{\prime} \\
& \lesssim \int_{\tau_{0}}^{\tau_{1}} \frac{\frac{e^{-\left(\tau-\tau_{1}\right)}|z|^{2}}{4\left(1+e^{-\frac{1}{2}}\right)}}{\left(1-e^{-\left(\tau-\tau_{1}\right)}\right)^{\frac{n}{4}}}\left\|e^{A_{z}\left(\tau_{1}-\tau^{\prime}\right)} e^{-\tau^{\prime}} G_{\text {out }}^{\perp}\right\|_{\rho} d \tau^{\prime} \\
& \lesssim \int_{\tau_{0}}^{\tau_{1}} e^{-(l+1)\left(\tau_{1}-\tau^{\prime}\right)}\left\|e^{-\tau^{\prime}} G_{\text {out }}^{\perp}\right\|_{\rho} d \tau^{\prime} \\
& \lesssim \int_{\tau_{0}}^{\tau_{1}} e^{-(l+1)\left(\tau_{1}-\tau^{\prime}\right)} e^{-\left(2 l+\frac{1}{2}\right) \tau^{\prime}} d \tau^{\prime} \\
& \lesssim e^{-(l+1) \tau}
\end{aligned}
$$

We next estimate the second term. We apply Lemma 7.5 to get

$$
\int_{\tau_{1}}^{\tau} e^{A_{z}\left(\tau-\tau^{\prime}\right)} e^{-\tau^{\prime}}\left|G_{\text {out }}^{\perp}\right| d \tau^{\prime} \lesssim \frac{1}{R^{\frac{1}{4}}} \frac{e^{-l \tau_{1}}}{1+|y|^{\frac{1}{4}}}+T^{b} e^{-l \tau_{1}}+e^{-(2 l+1) \tau}|z|^{4 l+4}+e^{-\left(l+\frac{2 l+1}{2 l+2}\right) \tau}|z|^{2 l+2} .
$$

Since $|z|=e^{\frac{\tau-\tau_{1}}{2}}$, it holds that $e^{-l \tau_{1}}=e^{-l \tau}|z|^{2 l}$. Therefore we deduce that

$$
\begin{aligned}
\int_{\tau_{1}}^{\tau} e^{A_{z}\left(\tau-\tau^{\prime}\right)} e^{-\tau^{\prime}}\left|G_{\text {out }}^{\perp}\right| d \tau^{\prime} & \lesssim \frac{1}{R^{\frac{1}{4}}} \frac{e^{-l \tau}|z|^{2 l}}{1+|y|^{\frac{1}{4}}}+T^{b} e^{-l \tau}|z|^{2 l}+e^{-(2 l+1) \tau}|z|^{4 l+4}+e^{-\left(l+\frac{2 l+1}{2 l+2}\right) \tau}|z|^{2 l+2} \\
& \lesssim \frac{e^{-l \tau}}{R^{\frac{1}{4}}}|z|^{2 l}+e^{-(2 l+1) \tau}|z|^{4 l+4}+e^{-\left(l+\frac{2 l+1}{2 l+2}\right) \tau}|z|^{2 l+2} .
\end{aligned}
$$

The estimate for the last term is derived from Lemma 7.5 .

7.4.4 (iv) Estimate in $|z|>e^{\frac{\tau-\tau_{0}}{2}}$ and $\tau \in\left(\tau_{0}+1, \infty\right)$

We get from Lemma 7.5 that

$$
\begin{aligned}
\int_{\tau_{0}}^{\tau} e^{A_{z}\left(\tau-\tau^{\prime}\right)} e^{-\tau^{\prime}}\left|G_{\text {out }}^{\perp}\right| d \tau^{\prime}<c & \left(\frac{1}{R^{\frac{1}{4}}} \frac{e^{-l \tau_{0}}}{1+|y|^{\frac{1}{4}}}+T^{b} e^{-l \tau_{0}}+e^{-(2 l+1) \tau}|z|^{4 l+4}\right. \\
& \left.+e^{-\left(l+\frac{2 l+1}{2 l+2}\right) \tau}|z|^{2 l+2}\right) \quad \text { for }(z, \tau) \in \mathbb{R}^{5} \times\left(\tau_{1}, \infty\right) .
\end{aligned}
$$


Since $|z|>e^{\frac{\tau-\tau_{0}}{2}}$, it holds that $e^{-l \tau_{0}}<e^{-l \tau}|z|^{2 l}$. Therefore we obtain the desired estimate. Combining estimates (i) (iv), we complete the proof of Proposition 7.1 .

\subsection{Pointwise estimate for $\Phi(z, \tau)$ in $|z|>e^{\frac{l \tau}{2 l+2}}$}

We go back to the function $\varphi(z, \tau)$ defined in 7.2 .

Lemma 7.6. There exists $K_{4}>0$ independent of $R, \delta_{0}, \sigma$ such that

$$
|\varphi(z, \tau)|<K_{4} T^{\frac{l}{l+1}} \quad \text { for }|z|>e^{\frac{l \tau}{2 l+2}}, \tau>\tau_{0}
$$

Proof. We recall that

$$
\varphi(z, \tau)=\mathbf{b}(\tau) \cdot \mathbf{e}+\Phi_{1}(z, \tau)+\Phi_{2}(z, \tau)
$$

We now check that

$$
\bar{\varphi}(z, \tau)=K\left(2 e^{-\frac{l \tau_{0}}{l+1}}-e^{-\frac{l \tau}{l+1}}\right)
$$

gives a super solution in $|z|>e^{\frac{l \tau}{2 l+2}}$. From $(7.4)$, Lemma 7.2 and $(7.16)$, we note that

$$
|\varphi(z, \tau)| \lesssim e^{-(2 l+1) \tau}|z|^{2 l}+e^{-(l+1) \tau}|z|^{2 l+2}+e^{-l \tau}|z|^{2 l}+e^{-\left(l+\frac{2 l+1}{2 l+2}\right) \tau}|z|^{2 l+2}
$$

for $|z|>1$. Therefore we get

$$
|\varphi(z, \tau)| \lesssim e^{-\frac{l \tau}{l+1}} \quad \text { for }|z|=e^{\frac{l \tau}{2 l+2}} .
$$

Furthermore by 7.5 , we see that the initial data satisfies

$$
\begin{aligned}
\left|\varphi\left(\tau_{0}\right)\right| & =|\mathbf{d} \cdot \mathbf{e}| \chi_{\text {out }} \lesssim e^{-(2 l+1) \tau_{0}}\left(1+|z|^{2 l}\right) \mathbf{1}_{|z|<2 e^{\frac{\left(l+\frac{1}{2}\right) \tau_{0}}{2 l+2}}} \\
& \lesssim e^{-(2 l+1) \tau_{0}}\left(1+|z|^{2 l}\right) \mathbf{1}_{|z|<e^{\frac{\tau_{0}}{2}} \lesssim e^{-(l+1) \tau_{0}}}
\end{aligned}
$$

Finally we get from Lemma 7.1 that

$$
\begin{aligned}
e^{-\tau}\left|G_{\text {out }}\right| & \lesssim e^{-\left(l+\frac{2 l+1}{2 l+2}\right) \tau}|z|^{2 l+2} \mathbf{1} e^{\frac{\left(l+\frac{1}{2}\right) \tau}{2 l+2}}<|z|<2 e^{\frac{\left(l+\frac{1}{2}\right) \tau}{2 l+2}}+\delta_{0}^{2} e^{-\tau} \\
& \lesssim e^{-\frac{l \tau}{l+1}} \quad \text { for }|z|>e^{\frac{l \tau}{2 l+2}} .
\end{aligned}
$$

From this estimate, we verify that if $K \gg 1$

$$
\bar{\varphi}_{\tau}-A_{z} \bar{\varphi}=\frac{l K}{l+1} e^{-\frac{l \tau}{l+1}} \geq e^{-\tau}\left|G_{\text {out }}\right| \quad \text { for }|z|>e^{\frac{l \tau}{2 l+2}} .
$$

Therefore by a comparison argument, we obtain

$$
|\varphi(z, \tau)| \lesssim \bar{\varphi}(z, \tau) \lesssim K e^{-\frac{l \tau_{0}}{l+1}}=K T^{\frac{l}{l+1}} \quad \text { for }|z|>e^{\frac{l \tau}{2 l+2}}, \tau>\tau_{0}
$$

The proof is completed.

We now assume that

$$
T^{\frac{l}{l+1}}=e^{-\frac{l R}{l+1}}<\delta_{0}^{2}
$$

From this relation, Lemma 7.6 is rewritten as

$$
|\varphi(z, \tau)|<K_{4} \delta_{0}^{2} \quad \text { for }|z|>e^{\frac{l \tau}{2 l+2}}, \tau>\tau_{0}
$$

We finally derive estimates in $|x|>1$. Let $W(x, t)$ be a solution of 7.1 .

Lemma 7.7. There exists $K_{5}>0$ independent of $R, \delta_{0}, \sigma$ such that

$$
|W(x, t)|<K_{5}\left(\frac{T^{3 l+3}}{|x|^{3}}+\frac{\delta_{0}^{2}}{|x|^{2}}\right) \quad \text { for }|x|>1, t \in(0, T) .
$$


Proof. We now claim that

$$
\bar{W}(x, t)=\frac{K}{|x|^{3}}\left(T^{3 l+3}-(T-t)^{3 l+3}\right)+\frac{K \delta_{0}^{2}}{|x|^{2}}
$$

gives a super solution in $|x|>1$. Since $\varphi(z, \tau)=W(x, t)$ (see Section 7.1), from 7.17), we verify that if $K>K_{4}$

$$
|W(x, t)|<K_{4} \delta_{0}^{2} \leq \bar{W}(x, t) \quad \text { for }|x|=1 \text {. }
$$

Furthermore from Lemma 7.1 , we recall that

$$
\left|G_{\text {out }}\right| \lesssim \frac{(T-t)^{3 l+2}}{|x|^{3}}+\frac{\delta_{0}^{2}}{|x|^{2 p}} \quad \text { for }|x|>1
$$

Therefore since $p>2$, we get if $K \gg 1$

$$
\bar{W}_{t}-\Delta_{x} \bar{W}=\frac{(3 l+3) K}{|x|^{3}}(T-t)^{3 l+2}+\frac{2 K \delta_{0}^{2}}{|x|^{4}} \geq\left|G_{\text {out }}\right| \quad \text { for }|x|>1 .
$$

Since $W(x, t)=0$ for $|x|>1, t=0$ (see 7.1$)$, by a comparison argument, we conclude

$$
|W(x, t)|<\bar{W}(x, t) \quad \text { for }|x|>1, t \in(0, T) .
$$

This complete the proof.

\section{Proof of Theorem 1}

We perform the argument mentioned in Section 5.2 rigorously. We fix $\delta_{0} \in(0,1)$ small enough. Let $\tilde{w}(x, t) \in C\left(\mathbb{R}^{5} \times[0, T]\right)$ be an extension of $w(x, t) \in X_{\sigma}$ defined in Section 5.2. $(\lambda(t), \epsilon(y, t))$ be a pair of functions constructed in Section 6 , and $W(x, t)$ be a function obtained in Section 7. We now define

$$
w(x, t) \in X_{\sigma} \mapsto W(x, t) \in C\left(\mathbb{R}^{5} \times[0, T)\right) \cap C^{2,1}\left(\mathbb{R}^{5} \times(0, T)\right) .
$$

From Proposition 7.1 and Lemma 7.6- Lemma 7.7 there exists $R_{0}>0$ such that $W(x, t) \in X_{\sigma}$ if $R>R_{0}$. By the way of construction, the mapping $w(x, t) \in\left(X_{\sigma}, d_{X_{\sigma}}\right) \mapsto W(x, t) \in\left(X_{\sigma}, d_{X_{\sigma}}\right)$ is continuous, where $d_{X_{\sigma}}$ is the metric in $X_{\sigma}$ defined in (5.8). Furthermore we note that

$$
\frac{\alpha_{l}^{2}}{2}(2 \sigma)^{2 l+2} \lambda(t)<2 \alpha_{2}^{2} T^{2 l+2} \quad \text { and } \quad \tau_{0}<\tau<|\log (2 \sigma)| \quad \text { for } t \in(0, T-2 \sigma) .
$$

Therefore from the local Hölder estimate and the decay of space infinity $W(x, t)<\frac{\delta_{0}}{1+|x|^{2}}$ for $|x|>1$, we find that the mapping is compact. From the Schauder fixed point theorem, we obtain a function $w(x, t)=W(x, t) \in X_{\sigma}$, which is denoted by $w_{\sigma}(x, t)$. Since $\tilde{w}_{\sigma}(x, t)=w_{\sigma}(x, t)$ for $t \in(0, T-2 \sigma)$ (see Section 5.2), a pair of functions $\left(w_{\sigma}(x, t), \lambda_{\sigma}(t), \epsilon_{\sigma}(y, t)\right)$ gives a solution of 5.5 for $t \in(0, T-2 \sigma)$. We take $\sigma \rightarrow 0$ to obtain $w(x, t)=\lim _{\sigma \rightarrow 0} w_{\sigma}(x, t)$. This is the desired solution, which has the form

$$
u(x, t)=\mathrm{Q}_{\lambda}+\Theta(x, t) \chi_{\mathrm{out}}+\lambda^{-\frac{n-2}{2}} \epsilon(y, t) \chi_{\mathrm{in}}+w(x, t)
$$

with

$$
\left|\lambda(t)-\alpha_{l}^{2}(T-t)^{2 l+2}\right|<K\left(\delta_{0}+\frac{T-t}{R^{2}}\right)(T-t)^{2 l+2} .
$$

If we repeat the argument for any $A<0$ (we fixed $A=-1$ in Section 4 , we obtain the solution described in Theorem 1 .

\section{Acknowledgement}

The author is partly supported by Grant-in-Aid for Young Scientists (B) No. 26800065. 


\section{References}

[1] C. Collot, F. Merle, P. Raphaël, Dynamics near the ground state for the energy critical nonlinear heat equation in large dimensions, Comm. Math. Phys. 352 no.1 (2017) 215-285.

[2] C. Cortázar, M. del Pino, M. Musso, Green's function and infinite-time bubbling in the critical nonlinear heat equation, Preprint arXiv:1604.07117.

[3] J. Davila, M. del Pino, J. Wei, Singularity formation for the two-dimensional harmonic map flow into $S^{2}$, Preprint arXiv:1702.05801.

[4] M. del Pino, M. Musso, J. Wei, Infinite time blow-up for the 3-dimensional energy critical heat equation, Preprint arXiv:1705.01672.

[5] M. del Pino, M. Musso, J. Wei, Geometry driven Type II higher dimensional blow-up for the critical heat equation, Preprint arXiv:1710.11461.

[6] M. del Pino, M. Musso, J. Wei, Type II blow-up in the 5-dimensional energy critical heat equation, Preprint arXiv:1808.10637.

[7] S. Filippas, M. A. Herrero, J. J. L. Velázquez, Fast blow-up mechanisms for sign-changing solutions of a semilinear parabolic equation with critical nonlinearity, R. Soc. Lond. Proc. Ser. A Math. Phys. Eng. Sci. 456 no. 2004 (2000) 2957-2982.

[8] Y. Giga, R. Kohn, Characterizing blowup using similarity variables, Indiana Univ. Math. J. 36 no. 1 (1987) 1-40.

[9] J. Harada Blowup profile for a complex valued semilinear heat equation, J. Funct. Anal. 270 no. 11 (2016) 4213-4255.

[10] J. Harada Construction of type II blow-up solutions for a semilinear parabolic system with higher dimension, Calc. Var. Partial Differential Equations 56 no. 4 (2017) Art 121.

[11] M. A. Herrero, J. J. L. Velázquez, Explosion de solutions d'équations paraboliques semilinéaires supercritiques, C.R. Acad. Sci. Paris Sér. I 319 no. 2 (1994) 141-145.

[12] M. A. Herrero, J. J. L. Velázquez, A blow up result for semilinear heat equations in the supercritical case, unpublished.

[13] G. M. Lieberman, Second Order Parabolic Differential Equations, World Scientific, Singapore, 1996.

[14] H. Matano, F. Merle, On nonexistence of type II blowup for a supercritical nonlinear heat equation, Comm. Pure Appl. Math. 57 no. 11 (2004) 1494-1541.

[15] F. Merle, P. Raphaël, I. Rodnianski, Blowup dynamics for smooth data equivariant solutions to the critical Schrodinger map problem, Invent. Math. 193 no. 2 (2013) 249-365.

[16] N. Mizoguchi, Type-II blowup for a semilinear heat equation, Adv. Differential Equations 9 no. 11-12 (2004) 12791316.

[17] P. Raphaël, I. Rodnianski, Stable blow up dynamics for the critical co-rotational wave maps and equivariant YangMills problems, Publ. Math. Inst. Hautes Études Sci. 115 (2012) 1-122.

[18] Y. Seki, Type II blow-up mechanisms in a semilinear heat equation with critical Joseph-Lundgren exponent, J. Funct. Anal. 275 no. 12 (2018) 3380-3456.

[19] R. Schweyer, Type II blow-up for the four dimensional energy critical semi linear heat equation, J. Funct. Anal. 263 no. 12 (2012) 3922-3983. 